\title{
How the study of networks informs knowledge translation and implementation: a scoping review
}

\author{
Stephanie M. N. Glegg ${ }^{1,2,3^{*}}$ (D), Emily Jenkins ${ }^{4}$ and Anita Kothari ${ }^{5}$
}

\begin{abstract}
Background: To date, implementation science has focused largely on identifying the individual and organizational barriers, processes, and outcomes of knowledge translation (KT) (including implementation efforts). Social network analysis (SNA) has the potential to augment our understanding of KT success by applying a network lens that examines the influence of relationships and social structures on research use and intervention acceptability by health professionals. The purpose of this review was to comprehensively map the ways in which SNA methodologies have been applied to the study of KT with respect to health professional networks.

Methods: Systematic scoping review methodology involved searching five academic databases for primary research on $\mathrm{KT}$ that employed quantitative SNA methods, and inclusion screening using predetermined criteria. Data extraction included information on study aim, population, variables, network properties, theory use, and data collection methods. Descriptive statistics and chronology charting preceded theoretical analysis of findings.

Results: Twenty-seven retained articles describing 19 cross-sectional and 2 longitudinal studies reported on 28 structural properties, with degree centrality, tie characteristics (e.g., homophily, reciprocity), and whole network density being most frequent. Eleven studies examined physician-only networks, 9 focused on interprofessional networks, and 1 reported on a nurse practitioner network. Diffusion of innovation, social contagion, and social influence theories were most commonly applied.

Conclusions: Emerging interest in SNA for KT- and implementation-related research is evident. The included articles focused on individual level evidence-based decision-making: we recommend also applying SNA to meso- or macro-level $\mathrm{KT}$ activities. SNA research that expands the range of professions under study, examines network dynamics over time, extends the depth of analysis of the role of network structure on KT processes and outcomes, and employs mixed methods to triangulate findings, is needed to advance the field. SNA is a valuable approach for evaluating key network characteristics, structures and positions of relevance to $\mathrm{KT}$, implementation, and evidence informed practice. Examining how network structure influences connections and the implications of those holding prominent network positions can provide insights to improve network-based KT processes.
\end{abstract}

Keywords: Knowledge translation, Evidence-based practice, Social network analysis, Information flow, Scoping review, Network, Implementation

\footnotetext{
* Correspondence: sglegg@cw.bc.ca

${ }^{1}$ Rehabilitation Sciences, The University of British Columbia, 212 - 2177

Wesbrook Mall, Vancouver, BC V6T 1Z3, Canada

${ }^{2}$ Therapy Department, Sunny Hill Health Centre for Children, 3644 Slocan

Street, Vancouver, BC V5M 3E8, Canada

Full list of author information is available at the end of the article
}

(c) The Author(s). 2019 Open Access This article is distributed under the terms of the Creative Commons Attribution 4.0 International License (http://creativecommons.org/licenses/by/4.0/), which permits unrestricted use, distribution, and reproduction in any medium, provided you give appropriate credit to the original author(s) and the source, provide a link to the Creative Commons license, and indicate if changes were made. The Creative Commons Public Domain Dedication waiver (http://creativecommons.org/publicdomain/zero/1.0/) applies to the data made available in this article, unless otherwise stated. 


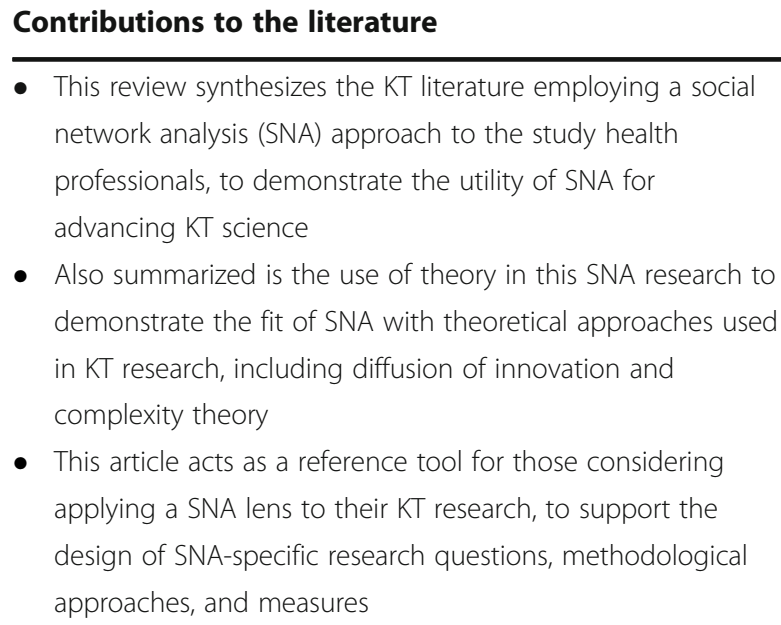

- This review synthesizes the KT literature employing a social network analysis (SNA) approach to the study health professionals, to demonstrate the utility of SNA for advancing KT science

- Also summarized is the use of theory in this SNA research to demonstrate the fit of SNA with theoretical approaches used in KT research, including diffusion of innovation and complexity theory

- This article acts as a reference tool for those considering applying a SNA lens to their KT research, to support the design of SNA-specific research questions, methodological approaches, and measures

\section{Background}

The "science" of knowledge translation (KT), namely the study of the processes, determinants, and outcomes of $\mathrm{KT}$ and evidence informed practice (EIP) efforts [1], is of high interest to researchers because of the significant challenges that exist in getting research into practice. To date, KT scientists have targeted their efforts primarily at identifying the key processes of $\mathrm{KT}$, understanding the influences on these processes, and evaluating the effectiveness of various strategies to support them [2-5]. However, identifying the specific mechanisms by which KT strategies, particularly complex multi-faceted ones, have been effective requires additional research. Furthermore, limited information is available to clinicians and researchers about the role of social relationships and network connections in facilitating KT [6].

Social network analysis (SNA) is a research paradigm concerned with the patterns of connections (i.e., ties) between actors (i.e., people or entities) within an interconnected group or network, and how this "social structure" impacts outcomes of interest [7]. Key SNA terms that provide the context for this review are defined in Table 1, along with their application to the study of KT. Actors may be individuals, organizations, countries, or other entities; ties reflect the connections or linkages between them [7]. The structural characteristics of both whole and individuals' networks can be studied. Included with the paradigm are theories, such as network, graph, diffusion, and social influence theories, and a set of methodologies that can be applied across a range of substantive problems. Visualization, or mapping, and the use of network descriptive statistics can provide a visual and empirical basis for comparison across networks, including identifying important strengths, gaps, or differences between networks that merit further exploration through qualitative means. Statistical and computational modeling can also be used to explain and to predict network-related phenomena, and to simulate the complexities inherent in network dynamics.

SNA offers an alternate perspective to behavior change theory-based approaches prevalent in KT science [5]. These latter approaches focus on individual-level factors influencing behavior change, often from a social cognition perspective [5]. Conversely, SNA proposes a network-level perspective that examines how connections among individuals or entities, and the nature of the associated interactions, influence an outcome (e.g., accessing or sharing evidence, changing practice behaviors based on evidence). The paradigm respects the socially driven nature of innovation uptake, and the value inherent in examining not only the processes involved in $\mathrm{KT}$ but also the social structures and characteristics of the networks of relationships within which KT occurs. Examples of network-related KT processes that can be examined from a SNA lens include one-way versus two-way exchange of information, the timing and prediction of evidence uptake by different types of individuals, the influence of specific types of people on behavior change, individuals' capacity for change based on their positions in the network, gaps in the flow of or access to information or resources required for evidence use, and testing the effectiveness of strategies to address gaps or inefficiencies identified in the network.

Recent systematic reviews on SNA in health care have focused on quality and patient safety initiatives $[8,9]$, on a single profession (i.e., nursing) $[10,11]$, or on only select network properties (e.g., the study of brokers) [12]. Some reviews focus on conditions (e.g., obesity networks) [12] to explore possible network interactions for potential treatments. Given the complex and interprofessional nature of health care practice, a study of the full breadth of health professions and network properties is required. Furthermore, some of these reviews included non-health care literature (e.g., from television production and corporate business contexts) [13], or neglected to include social sciences databases in which most SNA journals are indexed. The existing broad reviews of health professional networks $[6,14,15]$ do include some studies on KT-related phenomena (e.g., diffusion, knowledge transfer); however, the majority of their content centered on the study of social interactions that have implications for organizational functioning (e.g., friendships, work task assignments, staff recruitment, social support trust), but were not linked directly to 
Table 1 Social network analysis (SNA) terms and their implications for knowledge translation

\begin{tabular}{|c|c|c|}
\hline $\begin{array}{l}\text { SNA term (frequency } \\
\text { count) }\end{array}$ & Definition & Implication for KT \\
\hline Network & $\begin{array}{l}\text { An interconnected group of actors (e.g., people, } \\
\text { organizations) [7] }\end{array}$ & Provides the social context within which KT occurs \\
\hline Actor & $\begin{array}{l}\text { A point (node) in a network that represents an individual, } \\
\text { organization or entity connected to other actors (through } \\
\text { ties) [7] }\end{array}$ & $\begin{array}{l}\text { Represents the people, teams, or organizations involved } \\
\text { in KT processes }\end{array}$ \\
\hline Tie (2) & $\begin{array}{l}\text { The relations or connections among actors in the } \\
\text { network [79] }\end{array}$ & $\begin{array}{l}\text { Represents the interactions, collaborations, or } \\
\text { relationships involved in KT Measures one- versus two- } \\
\text { way communication, advice seeking, collaboration, etc. } \\
\text { [7] }\end{array}$ \\
\hline Dyad & Pairwise relations between actors [7] & $\begin{array}{l}\text { Represents one of three levels of analysis for social } \\
\text { network data (the others being individual node-level } \\
\text { and whole network level) [7] }\end{array}$ \\
\hline \multicolumn{3}{|l|}{ Centralization } \\
\hline $\begin{array}{l}\text { Whole network } \\
\text { centralization (3) }\end{array}$ & $\begin{array}{l}\text { Extent to which interconnections are unequal across the } \\
\text { network [21] (i.e., concentrated around one or more central } \\
\text { individuals) [7] }\end{array}$ & $\begin{array}{l}\text { Thought to enhance ease of knowledge sharing and to } \\
\text { promote standard practices of existing protocols [80]. } \\
\text { Decentralization may support new innovations, but } \\
\text { lead to mixed messaging and decreased clarity because } \\
\text { of multiple information sources [72] }\end{array}$ \\
\hline
\end{tabular}

\section{Centrality}

Degree centrality (3)

Indegree centrality (10)

Outdegree centrality (5)

Betweenness centrality

(4)

Flow betweenness centrality (3)

Closeness centrality (2)

Bonacich centrality (1)

Hubs and authorities centrality (1)

Tie characteristics

Tie strength (7)

Tie homophily (includes external-internal or El index) (13)

Tie hierarchy (1)

Tie reciprocity (8)

Euclidian distance (1)
\# of direct ties (connections) of an actor

\# of individuals who send (identify) ties to an actor

\# of direct ties an actor sends (identifies) to others [33]

Extent to which an individual is tied/connected to others who are not connected themselves [40]

How involved an actor is in all of the paths or routes between all other actors (not just those representing the shortest paths) [79]

Proportion of actors that can be reached in one or more steps [79]

Extent to which an actor is tied to others, weighted according to the centrality (e.g., popularity, importance) of those to whom the actor is tied/connected [79]

The structural prominence of individuals within a coreperiphery structured network [32]

Value associated with a tie/connection, e.g., frequency of contact, emotional intensity, duration of connection, etc. [7]

Similarity of connected actors/nodes on a given attribute [7]

Connections between actors dissimilar in their status (e.g., according to profession, leadership or power position) [7]

The extent to which directional ties to actors are reciprocated (i.e., are bi-directional) [79]

A measure of the dissimilarity between the tie patterns of each pair of actors in the network [79]
Seen as an indicator of visibility [81], prestige [39] or power [79] resulting from lots of direct contact to many others

Considered an index of importance [28] power or influence [40]

Used to quantify access to resources through colleagues, exposure to evidence and others' practices; positively associated with EIP use [33]

Used as a proxy for control of KT processes [39]; high values reflect a favorable position (e.g. brokering potential) [40] for information flow or power [79]

Used to determine contributions of individuals toward team decision-making; provides insights into structural hierarchy [33] Used as a proxy for ease of bypassing the core individuals in the network $[39,79]$

Proxy for degree of access to information [39] or efficiency in communicating with the network (relative reach) [7]

Proxy for power or hierarchy within a network; may help to identify network fragmentation/brokering opportunities [14]

Proxy for importance [32]

Weak ties thought to increase access to new information/opportunities; strong ties seen as required for innovation implementation [82]

Similarities among people create conditions for social contagion (individuals may be more likely to modify their behaviors/attitudes to match those around them) $[67,83]$

Hierarchy may be a barrier to innovation adoption (e.g., lack of interest from above/resistance from below [29]

Reciprocity may reflect greater stability or equality (versus hierarchy) [79]

Can be used to identify key people by their dissimilarity to others (e.g., who has the most research productivity 
Table 1 Social network analysis (SNA) terms and their implications for knowledge translation (Continued)

\begin{tabular}{|c|c|c|}
\hline $\begin{array}{l}\text { SNA term (frequency } \\
\text { count) }\end{array}$ & Definition & Implication for KT \\
\hline & & $\begin{array}{l}\text { relative to their connected peers) [28] (as a proxy of } \\
\text { influence) }\end{array}$ \\
\hline \multicolumn{3}{|l|}{ Density } \\
\hline $\begin{array}{l}\text { Whole network density } \\
\text { (8) }\end{array}$ & $\begin{array}{l}\text { An index of the proportion of existing ties relative to all } \\
\text { possible ties in a network [79] }\end{array}$ & $\begin{array}{l}\text { Proxy for efficiency of information flow [79], solidarity } \\
\text { [84], or cohesiveness within a network [21] }\end{array}$ \\
\hline \multicolumn{3}{|l|}{ Ego network density (2) } \\
\hline \multicolumn{3}{|l|}{ Subgroups } \\
\hline Components/isolates (3) & $\begin{array}{l}\text { Portions of the network that contain actors connected to one } \\
\text { another, but disconnected from actors of other subgroups } \\
\text { [79] }\end{array}$ & $\begin{array}{l}\text { Subgroups and isolates can be targeted to increase } \\
\text { connectedness, share information, or mobilize action }\end{array}$ \\
\hline Cliques (1) & $\begin{array}{l}\text { Maximum \# of actors who share all possible connections } \\
\text { among themselves [79] }\end{array}$ & $\begin{array}{l}\text { Can describe paths for fostering awareness and } \\
\text { adoption of interventions [23] }\end{array}$ \\
\hline Clusters (4) & Dense sets of connections in a network [79] & $\begin{array}{l}\text { Identifying attributes that influence clustering helps } \\
\text { understand KT-related behaviors, such as information } \\
\text { seeking (e.g., experts; same department) }[36,41]\end{array}$ \\
\hline \multicolumn{3}{|l|}{ Network roles and positions } \\
\hline Brokers (1) & $\begin{array}{l}\text { Actors holding bridging positions in a network (i.e., play a role } \\
\text { in connecting subgroups) [79] }\end{array}$ & $\begin{array}{l}\text { Can leverage brokers' positions for efficient KT by } \\
\text { leveraging their tie paths/connectedness }[36,37,79]\end{array}$ \\
\hline $\begin{array}{l}\text { Coreness/Core-periphery } \\
\text { index (2) }\end{array}$ & $\begin{array}{l}\text { The core of a network represents the maximally dense area of } \\
\text { connections, whereas the periphery represents (to the } \\
\text { maximum extent possible), the set of nodes without } \\
\text { connections within their group [79] }\end{array}$ & $\begin{array}{l}\text { Power/influence at the core [39]. The most active EIP } \\
\text { practitioners may be found at periphery [32] }\end{array}$ \\
\hline $\begin{array}{l}\text { Structural equivalence } \\
\text { (2) }\end{array}$ & $\begin{array}{l}\text { When two actors/nodes have the same relationships to all } \\
\text { other nodes in the network-they can be substituted without } \\
\text { altering the network [79] }\end{array}$ & $\begin{array}{l}\text { These positions may generate social pressure within a } \\
\text { network }[24,25]\end{array}$ \\
\hline $\begin{array}{l}\text { Structural holes/ } \\
\text { constraint (ego network) } \\
\text { (2) }\end{array}$ & $\begin{array}{l}\text { Structural holes: absent ties in a network that limit exchange } \\
\text { between actors; constraint: degree to which an actor is tied } \\
\text { to others who are themselves connected [79] }\end{array}$ & $\begin{array}{l}\text { Inequality among actors can be identified and targeted } \\
\text { through KT interventions; may have implications for EIP } \\
\text { adoption [31] (e.g., many ties may restrict one's actions/ } \\
\text { capacity) [79] }\end{array}$ \\
\hline
\end{tabular}

Transitivity/network closure (i.e., network structure related to triads)

Alternating k-stars (4) The tendency of actors to create ties [29]

Alternating k-triangles/ transitive triads and/or non-closure

structures (5)

Cyclic closure (1)

Alternating independent two-paths (2)
The extent to which sets of 3 actors form patterns of connections that create larger "clumps" within the network $[29,79]$

The tendency for transitive triads (sets of three actors in which two ties exist) to lead to reciprocal ties within that triad [27]

Assesses the conditions required for transitivity (i.e., ties that form between each pair of actors in a set of three actors) [29]
Used as an indicator of hubs within a network [37] or the tendency to share/exchange knowledge [29]

Assesses tendency to build relationships outside of one's local group-access to new knowledge [29]

Cyclic closure thought to reflect non- hierarchical knowledge exchange, which is more effortful to maintain and therefore less likely to be seen in knowledge sharing networks [27]

Can determine the extent to which actors tend to build small, closed, non-hierarchical connections that limit broader access to new information [29]

SNA social network analysis, $K T$ knowledge translation

the exchange or application of evidence to inform practice. Similarly, the emphasis on outcomes related to work satisfaction, leadership roles, professional behaviors, protocol efficiency, patient flow, operating room layout, technology adoption, and workplace performance reduce the extent to which $\mathrm{KT}$-specific outcomes can be explored. The review by Chambers et al.
[6] described primarily the settings and outcomes of these studies, whereas the current study aims to describe in detail the nature of the application of SNA to the study of KT. Such an approach aims to advance the science of KT by providing insight into worthwhile methodological directions this literature can provide. Evidence for the effectiveness of specific KT 
interventions or for the identified relationships between network properties and other variables relevant to KT can be sought elsewhere. Furthermore, none of these reviews examined the use of theory in their included body of literature specifically, despite this focus being an identified gap [6]. Given the rapid growth of the use of SNA in the health care context over the past 8 years (see Fig. 2), an updated and more directed search is warranted. A targeted examination of the research specific to SNA in KT and EIP is required to inform the application of SNA methodologies in this field, with attention paid to the insights offered by both the structural properties examined and the theoretical perspectives applied.

A SNA perspective can broaden our understanding of the mechanisms by which KT efforts are effective by examining the social structures and relationships that facilitate or hinder KT and EIP. This understanding will augment our knowledge base by expanding the range of KT determinants worthy of consideration. As researchers gain interest in the social drivers of $\mathrm{KT}$ and EIP, this review will provide a foundation for developing key research questions and SNA-driven methodological approaches for KT research that are based on established and relevant theories. Furthermore, this review will add to the current theorizing in the field related to a systems-focused understanding of KT and implementation processes. Specifically, implementation happens within a complex system, and network approaches have been used to study complex systems; the link between the two areas demands greater attention. Given these gaps, the purpose of this article is to synthesize the ways in which SNA methodology can be used to advance the science of KT.

\section{Methods}

The purposes of a scoping review are to examine the extent, range, and nature of research in a given field; to determine the utility of conducting a subsequent systematic review; to summarize a body of research; and/ or to identify research gaps, making it an ideal approach to map the KT literature utilizing SNA [16]. The specific objectives of this scoping review were (1) to describe the literature on SNA as it has been applied to KT and EIP involving health care professionals, in terms of its research design, methodology, and key findings; (2) to provide a critical analysis of the results in the context of existing theory; and (3) to identify strengths and gaps to inform future research. The scoping methodology, as described by Levac, et al.'s [17] modification of Arksey and O'Malley's [16] guidelines, was applied. Step six in the methodology, consultation with key stakeholders, is optional and was not applied in the current review.

\section{Step 1: identify the research question}

The specific research questions developed for this review were:

1. How has SNA been applied to health professional networks in the field of KT/EIP with respect to study aims, data collection and analysis methods, and populations, context, variables, and structural properties under study?

2. What are the primary theoretical underpinnings that explain the link between the network properties and KT/EIP?

3. What are the gaps in the literature that can inform future research directions?

\section{Step 2: identify relevant literature}

The search strategy involved a systematic search of peer-reviewed English SNA literature in November 2015, repeated in July 2018, within five primary literature databases: MEDLINE, Cumulative Index to Nursing and Allied Health Literature (CINAHL), Embase, Web of Science (Science, Social Sciences Citation, and Arts and Humanities Citation Indexes), and Sociological Abstracts. Wherever possible, keywords were mapped to subject headings, which were focused to narrow the search (e.g., SNA terms) and exploded to broaden the search's scope (e.g., health care professional-related terms) to best capture relevant articles. Keywords encompassed concepts related to social networks, KT, implementation and EIP, as well as health care professionals. Because of the lack of consistent indexing terms addressing the concept of KT [18], keywords and subject headings were drawn from empirically evaluated search strategies on this topic to foster relevant results [18-20]. The Appendix provides the detailed search strategy for MEDLINE; other database strategies are available on request.

\section{Step 3: select the literature}

Retrieved articles were screened for inclusion by two authors (SG and EJ). Inclusion criteria included peer-reviewed articles describing outcomes of research studies employing quantitative SNA methodology to examine networks involving health care professionals in the context of KT, implementation, or EIP (broadly defined as the exchange and/or application of information to facilitate best practices in health care). The health professional context was selected to narrow the scope of the review while maintaining high relevance to $\mathrm{KT}$, as health professionals are common knowledge users or subjects of implementation efforts. Outcomes of interest included, but were not limited to competencies (i.e., attitudes, knowledge, or skills) and behaviors by health professionals related to their sharing or use of evidence to 
inform clinical decision-making. Dyadic (i.e., pair-level), ego-network (i.e., individuals' networks), and whole network (e.g., departmental or organizational-level) properties and variables were of interest. Exclusion criteria included non-English articles for feasibility, and articles that did not quantify SNA data or network properties to focus on articles that described, predicted, or explained network-related phenomena in the context of KT in quantitative terms specific to SNA (e.g., empirical studies whose analysis employed network data and analysis methods, as opposed to discussion papers). To target the scope toward evidence use by health professionals (i.e., to maintain relevance to $\mathrm{KT}$ involving health professionals within health care organizations), articles were excluded if they focused on online or social media-based networks (e.g., virtual communities of practice), policy-level KT, use of research by patients, focused on communication not explicitly involving research evidence or clinical decision-making about care based on evidence, or focused on the implementation of non-clinical interventions (e.g., electronic medical records, non-research-related quality improvement initiatives).

\section{Step 4: chart the data}

SG extracted the data using a structured table developed a priori in accordance with the research questions. Information from each study was captured with respect to study aim, population, sample size, variables, KT process and structural properties examined, theoretical perspective and data collection, and analysis methods employed. A second reviewer (AK) screened the extracted data for accuracy.

\section{Step 5: collate, summarize, and report results}

Descriptive statistics, including frequency counts and percentages, were calculated to provide an overview of the literature's breadth. Articles were charted by year of publication and country of first author to illustrate the chronological and geographical development of the field. Each network property identified in the reviewed articles

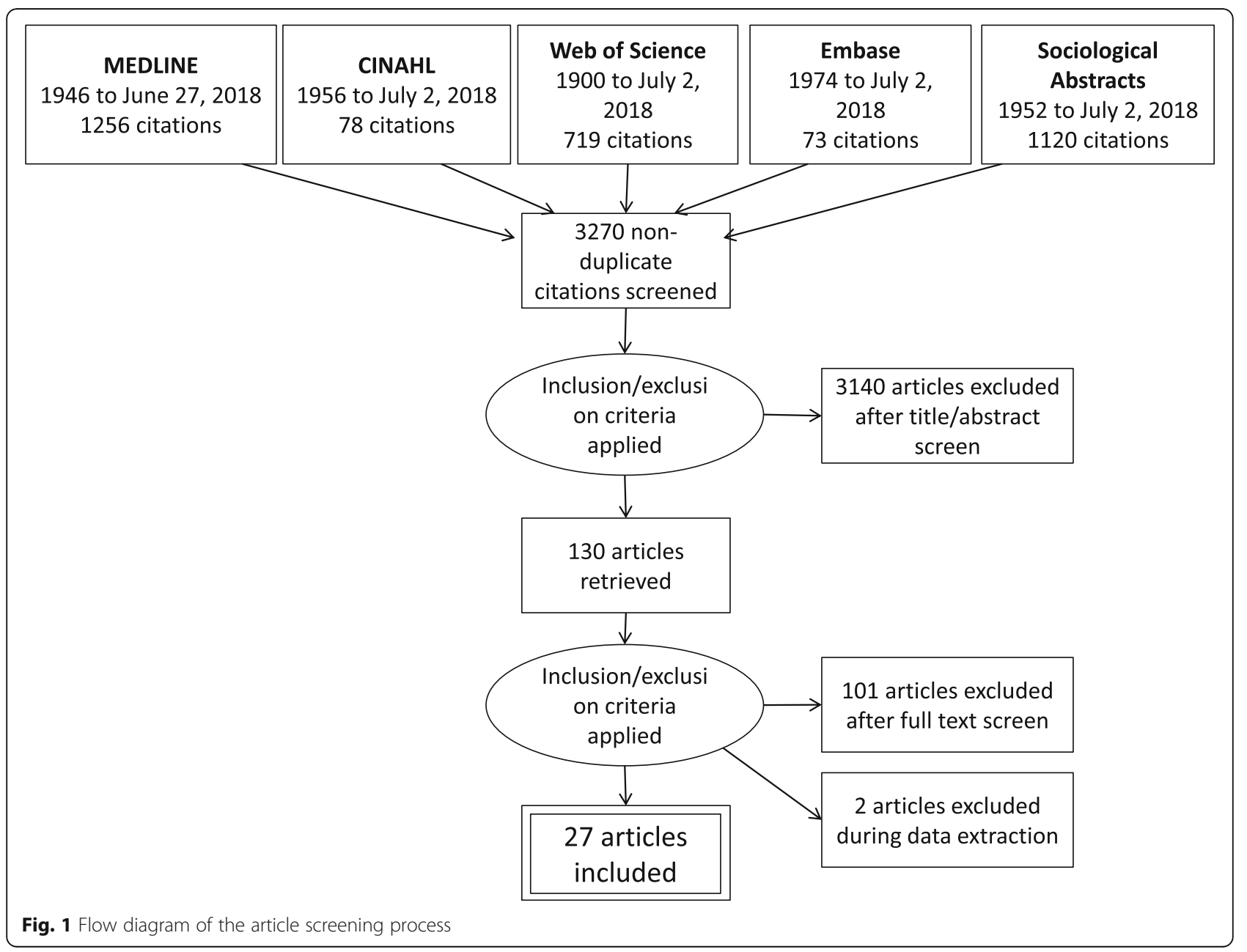




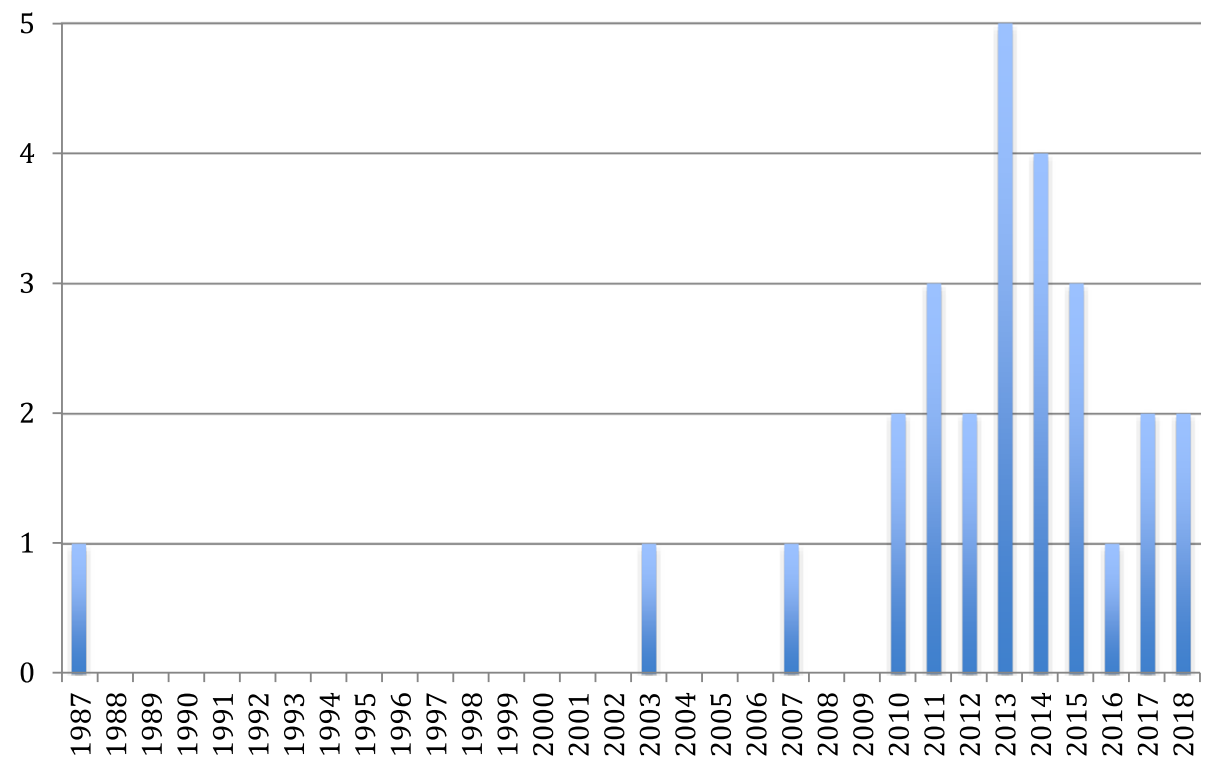

Fig. 2 Publications by year

is presented with respect to the relational parameter it represents in the context of studying KT. Key findings related to network properties were compiled and summarized narratively. An analysis of the use of SNA and the theory that informed this body of research was then performed.

\section{Results}

\section{Publication characteristics}

A total of 3531 articles were retrieved, of which 27 met inclusion criteria. Figure 1 shows the PRISMA flow diagram of included and excluded studies. Figure 2 depicts the frequency of publications by year. The USA and Italy (eight each) led in frequency, with Canada (4), Australia (2), the Netherlands (2), the UK (2), and Sweden (1) following. Study characteristics are presented in Table 2. Tallies and proportions of these studies presented in the following paragraphs do not sum to $100 \%$ in cases where the categories of characteristics are not mutually exclusive (e.g., a study may employ visualization of network properties while also presenting descriptive network property values).

\section{Study design and data collection}

The 21 studies' data sets were described in 27 articles, all but 2 of which employed cross-sectional designs yielding data at single time-points. Seventeen (81\%) of the studies collected SNA data through surveys, 2 (10\%) using a survey alongside interviews to support SNA data interpretation [21, 22], and $1(5 \%)$ through telephone interviews [23]. Two (10\%) studies employed document review (i.e., prescription, referral, or intervention records); one for SNA data collection and one to support outcome measurement [24-26].

\section{Networks and actors}

Physician-only networks were the most commonly studied $(11,52 \%)$ [23-25, 27-35], followed by interprofessional networks of researchers and clinicians (6, $29 \%)$ [36-41]. Only one study (5\%) examined the network of nurses and physicians [22], and one a network of nurse practitioners [42]. Two of the interprofessional networks included public health officials [36-38], one included leadership (i.e., directors, managers) and administrative support personnel [36, 37], and one included leadership and knowledge brokers [39]. In some cases, interprofessional network members' professions or formal roles within the network were not clearly indicated [21, 38, 39]. For two studies, the analysis of a subset of network members (i.e., managers/professional consultants; physicians with specific clinical workloads) was carried out $[35,40]$.

Studies examined networks ranging in size from 13 to 784 participants, with a mean of 153 . Just over half the studies (12, 57\%) were conducted across organizational boundaries [23-28, 30-35, 43]. Eight (38\%) were conducted within a single health care organization $[21,22,29,36,37,39,42,44$, $45]$, one (5\%) was conducted within a research-focused network [40, 41], and one (5\%) within a health-specific field at a national level [38]. 
Table 2 Characteristics of included studies

\begin{tabular}{|c|c|c|c|c|c|}
\hline Citation & Study purpose & Type of network/setting & $\begin{array}{l}\text { Network size (\# } \\
\text { participants) }\end{array}$ & $\begin{array}{l}\text { Data } \\
\text { collection } \\
\text { methods }\end{array}$ & $\begin{array}{l}\text { Theoretical } \\
\text { perspective }\end{array}$ \\
\hline Zappa 2011 [29] & $\begin{array}{l}\text { To describe relationships for } \\
\text { knowledge sharing about a } \\
\text { new drug }\end{array}$ & $\begin{array}{l}\text { Physician network within a } \\
\text { group of } 338 \text { hospitals }\end{array}$ & 784 physicians & Survey & $\begin{array}{l}\text { Diffusion of } \\
\text { innovation }\end{array}$ \\
\hline $\begin{array}{l}\text { Yousefi-Nooraie } \\
2014[37] \text { (same } \\
\text { study as 29) }\end{array}$ & $\begin{array}{l}\text { To assess factors associated } \\
\text { with information seeking in } \\
\text { public health }\end{array}$ & $\begin{array}{l}\text { Information seeking, expertise } \\
\text { recognition, and friendship } \\
\text { networks within an urban } \\
\text { public health department }\end{array}$ & $\begin{array}{l}15 \text { managers and } 13 \\
\text { professional consultants ( } n \\
=28 \text { ) }\end{array}$ & Survey & $\begin{array}{l}\text { Transactive } \\
\text { memory theory; } \\
\text { social exchange } \\
\text { theory }\end{array}$ \\
\hline $\begin{array}{l}\text { Yousefi-Nooraie } \\
2012[36] \text { (same } \\
\text { study as 30) }\end{array}$ & $\begin{array}{l}\text { To identify the structure of } \\
\text { intra-organizational knowledge } \\
\text { flow for evidence informed } \\
\text { practice }\end{array}$ & Information sharing network & $\begin{array}{l}170 \text { directors, managers, } \\
\text { supervisors, consultants, } \\
\text { epidemiologists, } \\
\text { practitioners, and } \\
\text { administrative support }\end{array}$ & & $\begin{array}{l}\text { Social influence } \\
\text { theory }\end{array}$ \\
\hline Tasselli 2015 [22] & $\begin{array}{l}\text { To describe knowledge } \\
\text { transfer between professions, } \\
\text { effectiveness of central actors }\end{array}$ & $\begin{array}{l}\text { Knowledge transfer network in } \\
\text { a hospital department }\end{array}$ & $\begin{array}{l}n=118 \\
53 \text { physicians and } 65 \\
\text { nurses }\end{array}$ & $\begin{array}{l}\text { Survey and } \\
\text { interviews }\end{array}$ & $\begin{array}{l}\text { Sociology of } \\
\text { professions theory; } \\
\text { SNA paradigm }\end{array}$ \\
\hline
\end{tabular}

Sibbald 2013 [21] To explore patterns of Information seeking and information exchange among colleagues in inter-professional teams

Racko 2018 [47] To examine the influence of social position on knowledge exchange over time

Quinlan 2013 [42] To explore mechanisms of information sharing across professional boundaries

Paul 2015 [35] (portion of data from study [45])

To test a model examining the role of triadic dependence on reciprocity and homophily

Menchik 2017 [44] To explore the type of knowledge valued by physicians and the influence of networks, within six hospitals hospital prestige on evidenceseeking behavior and perceived esteem by peers

Mascia 2018 [27] To explore theoretical mechanisms explaining network formation across clinical sectors

Mascia 2014 [33] To explore the association between connectedness with colleagues and frequency of evidence use within a physician network

Mascia 2015 [48] (same data as study [30, 32]

Mascia 2011a [30] To determine the association (same data as study $[32,48]$
To explore the influence of homophily on tie formation between attitudes toward EIP and network structure and to identify predictors of sharing networks within six interdisciplinary primary health care teams

Knowledge exchange networks within three academic-clinical KT programs

Knowledge contribution to decision-making by members within multidisciplinary primary healthcare teams (two clinical decisions, so two networks for each of four clinical teams)

Influence network

Patient care network

Information seeking and clinical case discussion

$n=28$ (nurses, physicians, residents, allied health

Survey and semi-

structured dietician, social worker); interviews two sites: $n_{1}=19$ and $n_{2}=$ 8.

three surveys: $n_{1}=66 ; n_{2}=$ Surveys 70; $n_{3}=42$ clinicians and academics

Nurse practitioners $(n=13$ Online or fewer) survey

Advice-seeking networks within two regional health authorities

Collaboration networks within 5 health authorities

IP advice-sharing networks within 6 hospitals
104 pediatricians

Survey

$\begin{array}{lll}33 \text { physicians } & \text { Surveys } & \text { SNA paradigm } \\ 135 \text { physicians } & & \\ 126 \text { physicians } & \text { Survey } & \begin{array}{l}\text { Social influence } \\ \text { theory }\end{array}\end{array}$

97 pediatricians

Survey

Balance theory; structural holes perspective; homophily principle

Diffusion of innovation; social influence theory; social contagion; strength of weak ties [82]

\section{7 physicians}

Survey
Homophily principle 
Table 2 Characteristics of included studies (Continued)

\begin{tabular}{|c|c|c|c|c|c|}
\hline Citation & Study purpose & Type of network/setting & $\begin{array}{l}\text { Network size (\# } \\
\text { participants) }\end{array}$ & $\begin{array}{l}\text { Data } \\
\text { collection } \\
\text { methods }\end{array}$ & $\begin{array}{l}\text { Theoretical } \\
\text { perspective }\end{array}$ \\
\hline & collaborative ties & & & & \\
\hline $\begin{array}{l}\text { Mascia } 2013 \text { [32] } \\
\text { (same data as } \\
\text { study }[30,48] \text { ) }\end{array}$ & $\begin{array}{l}\text { To explore the relationship } \\
\text { between attitudes toward EIP } \\
\text { and network position }\end{array}$ & & & & $\begin{array}{l}\text { Core/periphery } \\
\text { model; structural } \\
\text { holes }\end{array}$ \\
\hline $\begin{array}{l}\text { Mascia 2011b [31] } \\
\text { (same study as } \\
[30,32,48])\end{array}$ & $\begin{array}{l}\text { To explore the association } \\
\text { between network structure } \\
\text { and propensity to adopt EIP }\end{array}$ & & 207 physicians & & $\begin{array}{l}\text { Social contagion; } \\
\text { structural holes } \\
\text { perspective }\end{array}$ \\
\hline $\begin{array}{l}\text { Long } 2014 \text { [41] } \\
\text { (same data as } \\
\text { study [40]) }\end{array}$ & $\begin{array}{l}\text { To examine the influence of } \\
\text { clustering on past, present, } \\
\text { and future collaborations } \\
\text { within a translational research } \\
\text { network }\end{array}$ & $\begin{array}{l}\text { Past, present, and intended } \\
\text { collaboration networks within } \\
\text { a research network }\end{array}$ & $\begin{array}{l}68 \text { researchers and } \\
\text { clinicians }\end{array}$ & $\begin{array}{l}\text { Online } \\
\text { survey }\end{array}$ & SNA paradigm \\
\hline $\begin{array}{l}\text { Long } 2013 \text { [40] } \\
\text { (same data as } \\
\text { study [41]) }\end{array}$ & $\begin{array}{l}\text { To identify key players within a } \\
\text { research network, their } \\
\text { common attributes, and their } \\
\text { perceived influence, power, } \\
\text { and connectedness }\end{array}$ & $\begin{array}{l}\text { Research collaboration and } \\
\text { dissemination networks within } \\
\text { a research network }\end{array}$ & & & \\
\hline Keating 2007 [45] & $\begin{array}{l}\text { To describe the network of } \\
\text { influential discussions among } \\
\text { physicians and to predict } \\
\text { network position }\end{array}$ & $\begin{array}{l}\text { Frequency of influential } \\
\text { conversations relevant to } \\
\text { practice within primary care }\end{array}$ & 38 physicians & Survey & SNA paradigm \\
\hline $\begin{array}{l}\text { Heijmans } \\
2017[26]\end{array}$ & $\begin{array}{l}\text { To explore relationships } \\
\text { between network properties } \\
\text { and quality of care }\end{array}$ & $\begin{array}{l}\text { Information exchange } \\
\text { networks within } 31 \text { general } \\
\text { practices }\end{array}$ & $\begin{array}{l}180 \text { health professionals } \\
\text { (physicians, residents, } \\
\text { nurses, pharmacy } \\
\text { assistants, social workers) }\end{array}$ & $\begin{array}{l}\text { Survey } \\
\text { document } \\
\text { review (i.e., } \\
\text { intervention } \\
\text { and referral } \\
\text { charting) }\end{array}$ & SNA paradigm \\
\hline $\begin{array}{l}\text { Guldbrandsson } \\
2012[38]\end{array}$ & $\begin{array}{l}\text { To identify potential national } \\
\text { opinion leaders in child health } \\
\text { promotion }\end{array}$ & $\begin{array}{l}\text { Discussion network within } \\
\text { national child health } \\
\text { promotion context }\end{array}$ & $\begin{array}{l}153 \text { researchers, public } \\
\text { health officials, } \\
\text { pediatricians and other } \\
\text { individuals }\end{array}$ & $\begin{array}{l}\text { Emailed } \\
\text { survey item }\end{array}$ & $\begin{array}{l}\text { Diffusion of } \\
\text { innovation }\end{array}$ \\
\hline Friedkin 2010 [25] & $\begin{array}{l}\text { To examine the association } \\
\text { between discussion networks, } \\
\text { marketing, and physician } \\
\text { prescribing practices }\end{array}$ & \multirow{2}{*}{$\begin{array}{l}\text { Advice and discussion } \\
\text { networks of physicians (re- } \\
\text { analysis of Coleman, Katz and } \\
\text { Menzel, } 1966 \text { historical data on } \\
\text { medication adoption) }\end{array}$} & \multirow[t]{2}{*}{125 physicians } & \multirow[t]{2}{*}{$\begin{array}{l}\text { Document } \\
\text { review (i.e., } \\
\text { prescription } \\
\text { records of } \\
\text { pharmacies) }\end{array}$} & \multirow{2}{*}{$\begin{array}{l}\text { Diffusion of } \\
\text { innovation; social } \\
\text { contagion; } \\
\text { cohesion; } \\
\text { structural } \\
\text { equivalence }\end{array}$} \\
\hline Burt 1987 [24] & $\begin{array}{l}\text { To test social contagion theory } \\
\text { by examining cohesion versus } \\
\text { structural equivalence as } \\
\text { drivers of tie formation }\end{array}$ & & & & \\
\hline Doumit 2014 [34] & $\begin{array}{l}\text { To identify opinion leaders and } \\
\text { their impact on EIP }\end{array}$ & $\begin{array}{l}\text { Advice networks of craniofacial } \\
\text { surgeons within } 14 \text { countries }\end{array}$ & 59 craniofacial surgeons & $\begin{array}{l}\text { Online } \\
\text { survey }\end{array}$ & $\begin{array}{l}\text { Diffusion of } \\
\text { innovation }\end{array}$ \\
\hline $\begin{array}{l}\text { Di Vincenzo } \\
2017 \text { [28] }\end{array}$ & $\begin{array}{l}\text { To explain the impact of } \\
\text { research productivity on tie } \\
\text { redundancy (i.e., connections } \\
\text { that lead to the same people/ } \\
\text { information) }\end{array}$ & $\begin{array}{l}\text { Advice seeking networks } \\
\text { within and external to a health } \\
\text { authority containing } 6 \\
\text { hospitals }\end{array}$ & 228 physicians & Survey & $\begin{array}{l}\text { Structural holes } \\
\text { perspective; } \\
\text { homophily } \\
\text { perspective }\end{array}$ \\
\hline Bunger 2016 [43] & $\begin{array}{l}\text { To evaluate change in advice } \\
\text { ego-network composition and } \\
\text { its impact on whole network } \\
\text { structure following implemen- } \\
\text { tation of a "learning collabora- } \\
\text { tive" model in improve care } \\
\text { quality }\end{array}$ & $\begin{array}{l}\text { Advice networks of clinicians } \\
\text { (psychologists, social workers, } \\
\text { others) and leadership in } 32 \\
\text { behavioral health agencies }\end{array}$ & $\begin{array}{l}132 \text { clinicians, supervisors } \\
\text { and senior leaders }\end{array}$ & Surveys & SNA paradigm \\
\hline Ankem 2003 [23] & $\begin{array}{l}\text { To understand communication } \\
\text { flow and its influence on } \\
\text { awareness/adoption of a }\end{array}$ & $\begin{array}{l}\text { Frequent discussion networks } \\
\text { within a sample drawn from } \\
\text { an online physician directory }\end{array}$ & $\begin{array}{l}32 \text { interventional } \\
\text { radiologists }\end{array}$ & $\begin{array}{l}\text { Phone } \\
\text { interviews }\end{array}$ & $\begin{array}{l}\text { Diffusion of } \\
\text { innovation; SNA } \\
\text { paradigm }\end{array}$ \\
\hline
\end{tabular}


Table 2 Characteristics of included studies (Continued)

\begin{tabular}{|c|c|c|c|c|c|}
\hline Citation & Study purpose & Type of network/setting & $\begin{array}{l}\text { Network size (\# } \\
\text { participants) }\end{array}$ & $\begin{array}{l}\text { Data } \\
\text { collection } \\
\text { methods }\end{array}$ & $\begin{array}{l}\text { Theoretical } \\
\text { perspective }\end{array}$ \\
\hline $\begin{array}{l}\text { D'Andreta } \\
2013 \text { [39] }\end{array}$ & $\begin{array}{l}\text { To compare the network } \\
\text { structures of three research/KT } \\
\text { program initiatives }\end{array}$ & $\begin{array}{l}\text { Informal advice giving and } \\
\text { seeking networks within each } \\
\text { of three academic-clinical KT } \\
\text { programs }\end{array}$ & $\begin{array}{l}n=\sim 260 \text { (directors, } \\
\text { managers, program } \\
\text { leaders, knowledge } \\
\text { brokers, researchers, and } \\
\text { others unspecified) }\end{array}$ & $\begin{array}{l}\text { Online } \\
\text { survey }\end{array}$ & $\begin{array}{l}\text { SNA paradigm; } \\
\text { Epistemic } \\
\text { differences } \\
\text { perspective }\end{array}$ \\
\hline
\end{tabular}

SNA social network analysis, EIP evidence informed practice, $K T$ knowledge translation. Where indicated by the articles' authors, the dependent variable is designated using bold text

\section{Study purposes and data analysis methods}

A SNA perspective has been used to explore the patterns and efficiencies of information sharing within and across professions, to identify positions of influence and determine their effectiveness, to predict or to explain patterns of ties based on attributes or network structure, to compare the structural characteristics of different models of $\mathrm{KT}$, and to examine the relationship between network properties and EIP attitudes and behaviors. Using a longitudinal approach, SNA has been used to evaluate the influence of social structure on knowledge exchange over time, as well as to evaluate network change following a quality improvement intervention.

The majority of articles focused on information flow, while individuals' adoption of a clinical practice, involvement in collaborations that support KT or EIP, and evidence-informed group decision-making garnered less interest. No research was available on other KT activities, such as the processes involved in developing guidelines or other KT tools, adapting knowledge to the local context, assessing barriers to change, facilitating departmental- or organizational-level practice or service delivery changes (including de-implementation), monitoring evidence use, evaluating $\mathrm{KT}$ effectiveness, or sustaining change over time [46].

\section{Describing networks}

Eight (29\%) of the 27 articles described networks by deriving network properties from relational data $[21,22,30,36,39$, $40,42]$. Two articles (10\%) used conventional descriptive statistics (e.g., frequency counts, proportions) to describe social network data [34, 38]. Network visualizations illustrated the data in 13 (48\%) articles [22, 30, 32, 34-37, 40, 42-45]. Of these, ten articles presented whole network graphs (i.e., illustrations of the network structure), two presented network graphs of subgroups within the network, and one mapped whole networks within graphs that accounted for covariates. Network properties represented in the graphs included centrality (i.e., connectedness, brokers (i.e., bridgers)), core versus periphery structure (i.e., central areas of high connectivity versus peripheral areas of lower connectivity), and tie strength (e.g., frequency of contact); and attributes, such as gender, professional role, size of clinical practice, and division, department or team, and organization or site. Visualizations were used to depict network property configurations (i.e., illustrated the definitions of network properties) in two articles. Conventional charts (e.g., boxplots, scatterplots and bar, line, and area charts) were also used in six articles to visualize relationships between variables (e.g., network properties with one another, diffusion or adoption over time, centrality versus adoption timing, or receipt of useful information, percentage of ties by strength at different time points).

\section{Testing hypotheses (network modeling)}

Fourteen articles described the use of regression, including ordinary least squares $[24,28,32,44,47]$, ordinal logistic regression [33], multi-level modeling [25, 26, 37], P2 logistic regression [35, 45], linear regression [22], and MR-QAP analysis $[30,48]$. Regression was used to test theory about the cause of social structure $[24,25,28,35,48]$, to explain the impact of social structure on knowledge exchange behaviors [47], ease of knowledge transfer [22], receipt of useful knowledge [22], or quality of care [26], and to describe the influence of hospital prestige on evidence-seeking behavior and perceived esteem by peers [44]. Three articles reported paired $t$ tests or Wilcoxon ranks to evaluate differences between groups [22, 26] or time points [43], the latter of which also employed analysis of variance. Two articles described the use of the Chi-square test to examine associations between attributes and network position, or between two attributes $[23,40]$. Exponential random graph models were used in three instances to predict or to explain the formation of ties based on attributes and network structure [27, 29, 37], and a single study employed factor analysis to construct groupings of individuals based on frequency of information exchange [23]. No studies employed stochastic actor-based network modeling to examine network change over time.

Sample hypotheses relating to tie formation included predictors, such as homophily, existing ties (leading to reciprocity), and having a formal mechanism within the organization for interacting. Further hypothesis examples included that higher professional status would be associated with more knowledge exchange, tie homophily (i.e., 
Table 3 Variables, network properties and key findings

\begin{tabular}{|c|c|c|c|c|c|}
\hline \multirow[t]{2}{*}{ Citation } & \multirow{2}{*}{$\begin{array}{l}\text { Primary data } \\
\text { analysis } \\
\text { method }\end{array}$} & \multicolumn{3}{|l|}{ Variables of interest } & \multirow[t]{2}{*}{ Findings } \\
\hline & & Attributes & $\begin{array}{l}\text { Structural or } \\
\text { relational parameters }\end{array}$ & $\begin{array}{l}\text { Network property } \\
\text { used as proxy for } \\
\text { structural } \\
\text { parameter }\end{array}$ & \\
\hline \multicolumn{6}{|c|}{ Descriptive/exploratory studies } \\
\hline \multirow{6}{*}{$\begin{array}{l}\text { Yousefi- } \\
\text { Nooraie } 2012 \\
\text { [36] (same } \\
\text { study as [37]) }\end{array}$} & \multirow{6}{*}{$\begin{array}{l}\text { Deriving } \\
\text { network } \\
\text { properties to } \\
\text { describe the } \\
\text { network }\end{array}$} & \multirow[t]{6}{*}{-} & Connectedness & $\begin{array}{l}\text { Whole network } \\
\text { density }\end{array}$ & Low density (1.2\%) observed \\
\hline & & & $\begin{array}{l}\text { Information } \\
\text { exchange }\end{array}$ & Tie reciprocity & \multirow{5}{*}{$\begin{array}{l}\text { Head management division identified as central } \\
\text { cluster bridging organizational divisions, with } \\
\text { hierarchical information flow. } \\
\text { Expertise recognition and information seeking } \\
\text { clustered within divisions; friendships spanned } \\
\text { departments; } \\
\text { Friendship and expertise recognition predicted } \\
\text { information seeking ties; } \\
\text { Network-identified brokers should receive same } \\
\text { interventions and supports as formal brokers }\end{array}$} \\
\hline & & & Prestige (key actors) & Indegree centrality & \\
\hline & & & $\begin{array}{l}\text { Mediating power of } \\
\text { actors }\end{array}$ & $\begin{array}{l}\text { Betweenness } \\
\text { centrality }\end{array}$ & \\
\hline & & & $\begin{array}{l}\text { Subgroups of } \\
\text { connected actors }\end{array}$ & Clusters & \\
\hline & & & $\begin{array}{l}\text { Brokers (actors } \\
\text { connecting distinct } \\
\text { teams/clusters of } \\
\text { alters) }\end{array}$ & $\begin{array}{l}\text { Brokerage patterns } \\
\text { (measured by } \\
\text { which groups the } \\
\text { information source } \\
\text { amd its recipients } \\
\text { belonged) }\end{array}$ & \\
\hline \multirow[t]{2}{*}{$\begin{array}{l}\text { Sibbald } 2013 \\
\text { [21] }\end{array}$} & \multirow[t]{2}{*}{$\begin{array}{l}\text { Deriving } \\
\text { network } \\
\text { properties to } \\
\text { describe the } \\
\text { network }\end{array}$} & - & $\begin{array}{l}\text { Cohesiveness related } \\
\text { to giving and } \\
\text { seeking research- } \\
\text { related information }\end{array}$ & $\begin{array}{l}\text { Whole network } \\
\text { density }\end{array}$ & $\begin{array}{l}\text { Low density for information seeking and giving } \\
(7-12 \%) \text { observed; suggested these behaviors not } \\
\text { a central focus of the interprofessional relationships }\end{array}$ \\
\hline & & Profession & $\begin{array}{l}\text { Key players (prestige) } \\
\text { in giving and seeking } \\
\text { research information }\end{array}$ & Indegree centrality & $\begin{array}{l}\text { Medical residents prominent in knowledge } \\
\text { exchange; physician seen as primary implementer } \\
\text { of evidence; nurses as intermediaries between } \\
\text { physicians and support staff; allied health more } \\
\text { likely to draw information from external networks }\end{array}$ \\
\hline \multirow[t]{2}{*}{$\begin{array}{l}\text { Quinlan } 2013 \\
\text { [42] }\end{array}$} & \multirow{2}{*}{$\begin{array}{l}\text { Deriving } \\
\text { network } \\
\text { properties to } \\
\text { describe the } \\
\text { network }\end{array}$} & \multirow{2}{*}{$\begin{array}{l}\text { Profession tenure } \\
\text { of the team } \\
\text { Occupational } \\
\text { distance among } \\
\text { members } \\
\text { Number of team } \\
\text { members }\end{array}$} & \multirow{2}{*}{$\begin{array}{l}\text { Communicative } \\
\text { power (i.e., the } \\
\text { facilitation of mutual } \\
\text { understanding } \\
\text { among other team } \\
\text { members) }\end{array}$} & $\begin{array}{l}\text { Flow betweenness } \\
\text { centrality }\end{array}$ & \multirow{2}{*}{$\begin{array}{l}\text { True interprofessional decision-making attributed } \\
\text { to low structural hierarchy. Nurse practitioners } \\
\text { (in newly formed teams) and registered nurses } \\
\text { (in established teams) tended to have greatest } \\
\text { communicative power. Mutual understanding } \\
\text { and professions' involvement varied across clinical } \\
\text { decision-making episodes }\end{array}$} \\
\hline & & & & $\begin{array}{l}\text { Change in flow } \\
\text { betweenness } \\
\text { centrality between } \\
\text { clinical decisions }\end{array}$ & \\
\hline \multirow{7}{*}{$\begin{array}{l}\text { Long } 2014 \\
\text { [41] (same } \\
\text { study as [40]) }\end{array}$} & \multirow{7}{*}{$\begin{array}{l}\text { Descriptive } \\
\text { SNA; } \\
\text { correlations } \\
\text { among } \\
\text { specific } \\
\text { network } \\
\text { properties } \\
\text { and/or } \\
\text { attributes }\end{array}$} & \multirow{3}{*}{$\begin{array}{l}\text { Geographic } \\
\text { proximity } \\
\text { profession (e.g., } \\
\text { clinicians/ } \\
\text { researchers) }\end{array}$} & \multirow{3}{*}{$\begin{array}{l}\text { Grouping based on } \\
\text { similarity in attributes }\end{array}$} & \# components & \multirow{7}{*}{$\begin{array}{l}\text { Geographical proximity, professional homophily } \\
\text { associated with clustering (past collaborations); } \\
\text { geographical proximity and past collaborative ties } \\
\text { influenced current and future collaborations. } \\
\text { Intended future collaborations were more } \\
\text { interprofessional. } \\
\text { Network density varied across networks (current } \\
4 \% \text {, future } 27 \% \text {, past } 31 \% \text { ). } \\
\text { Weak ties and reputation associated with intention } \\
\text { for future collaboration; strong ties associated with } \\
\text { current collaborations. }\end{array}$} \\
\hline & & & & $\begin{array}{l}\text { External-internal } \\
(\text { E-I) indices based } \\
\text { on tie homophily }\end{array}$ & \\
\hline & & & & $\begin{array}{l}\text { Clustering } \\
\text { coefficients } \\
\text { (comparing ego- } \\
\text { and whole net- } \\
\text { work density) }\end{array}$ & \\
\hline & & - & $\begin{array}{l}\text { Past strong } \\
\text { collaborations; } \\
\text { Current or future } \\
\text { collaborations }\end{array}$ & $\begin{array}{l}\text { Past collaboration } \\
\text { network tie } \\
\text { strength; Current } \\
\text { or future } \\
\text { collaboration ties }\end{array}$ & \\
\hline & & $\begin{array}{l}\text { Previous } \\
\text { experience in the } \\
\text { field }\end{array}$ & $\begin{array}{l}\text { Current or future } \\
\text { collaborations }\end{array}$ & $\begin{array}{l}\text { Current or future } \\
\text { collaboration ties }\end{array}$ & \\
\hline & & $\begin{array}{l}\text { Actor's } \\
\text { reputation }\end{array}$ & Indirect contacts & $\begin{array}{l}\text { Future } \\
\text { collaboration } \\
\text { network tie } \\
\text { strength }\end{array}$ & \\
\hline & & & $\begin{array}{l}\text { Future intended } \\
\text { collaborations }\end{array}$ & $\begin{array}{l}\text { Future } \\
\text { collaboration ties }\end{array}$ & \\
\hline
\end{tabular}


Table 3 Variables, network properties and key findings (Continued)

\begin{tabular}{|c|c|c|c|c|c|}
\hline \multirow[t]{2}{*}{ Citation } & \multirow{2}{*}{$\begin{array}{l}\text { Primary data } \\
\text { analysis } \\
\text { method }\end{array}$} & \multicolumn{3}{|l|}{ Variables of interest } & \multirow[t]{2}{*}{ Findings } \\
\hline & & Attributes & $\begin{array}{l}\text { Structural or } \\
\text { relational parameters }\end{array}$ & $\begin{array}{l}\text { Network property } \\
\text { used as proxy for } \\
\text { structural } \\
\text { parameter }\end{array}$ & \\
\hline $\begin{array}{l}\text { Long } 2013 \\
\text { [40] (same } \\
\text { study as [41]) }\end{array}$ & $\begin{array}{l}\text { Chi square } \\
\text { analyses to } \\
\text { test for } \\
\text { association } \\
\text { between } \\
\text { attributes } \\
\text { and network } \\
\text { position (i.e., } \\
\text { key actor } \\
\text { status) }\end{array}$ & $\begin{array}{l}\text { Current } \\
\text { workplace } \\
\text { Gender } \\
\text { Membership in } \\
\text { other networks } \\
\text { Qualifications } \\
\text { Approach to } \\
\text { work within the } \\
\text { network }\end{array}$ & $\begin{array}{l}\text { Key actors (with } \\
\text { respect to power, } \\
\text { influence or } \\
\text { connectedness) }\end{array}$ & $\begin{array}{l}\text { Indegree centrality } \\
\text { Betweenness } \\
\text { centrality }\end{array}$ & $\begin{array}{l}\text { A manager, and specific researchers and } \\
\text { clinicians identified as key players. } \\
\text { Apart from expert status and valuing } \\
\text { adequate network resources, network-identified } \\
\text { central players and formal brokers had little in } \\
\text { common. } \\
\text { Planned interventions and support for formal } \\
\text { broker roles may be misdirected if not also } \\
\text { offered to network-perceived central players or } \\
\text { brokers. } \\
\text { Network members may be better able to } \\
\text { correctly identify central actors than formal } \\
\text { brokers }\end{array}$ \\
\hline $\begin{array}{l}\text { Guldbrandsson } \\
2012 \text { [38] }\end{array}$ & $\begin{array}{l}\text { Traditional } \\
\text { descriptive } \\
\text { statistics, e.g., } \\
\text { frequency } \\
\text { counts, } \\
\text { percentages }\end{array}$ & $\begin{array}{l}\text { Profession } \\
\text { Work } \\
\text { organization } \\
\text { Geographical } \\
\text { region }\end{array}$ & $\begin{array}{l}\text { Information seeking } \\
\text { about child health } \\
\text { promotion }\end{array}$ & $\begin{array}{l}\text { Tie homophily (\%) } \\
\text { Indegree centrality } \\
\text { (frequency of } \\
\text { being named) }\end{array}$ & $\begin{array}{l}\text { Organization and professional field were } \\
\text { shared in nearly half of all information } \\
\text { seeking ties }\end{array}$ \\
\hline \multirow[t]{3}{*}{$\begin{array}{l}\text { Doumit } 2014 \\
\text { [34] }\end{array}$} & \multirow{3}{*}{$\begin{array}{l}\text { Percentage } \\
\text { of people } \\
\text { nominating } \\
\text { an actor; } \\
\text { descriptive } \\
\text { statistics } \\
\text { (frequency } \\
\text { counts, } \\
\text { percentages) }\end{array}$} & - & $\begin{array}{l}\text { Influence by central } \\
\text { actors }\end{array}$ & $\begin{array}{l}\text { Degree } \\
\text { centralization }\end{array}$ & \multirow{3}{*}{$\begin{array}{l}\text { Six individuals with high credibility influenced } \\
85 \% \text { of the network, suggesting opinion leaders } \\
\text { have potential for supporting evidence use }\end{array}$} \\
\hline & & $\begin{array}{l}\text { Reasons for } \\
\text { change in } \\
\text { medical } \\
\text { approach } \\
\text { (proportions) }\end{array}$ & - & - & \\
\hline & & $\begin{array}{l}\text { Barriers to clinical } \\
\text { decision-making } \\
\text { (proportions) }\end{array}$ & - & - & \\
\hline \multirow[t]{5}{*}{$\begin{array}{l}\text { D'Andreta } \\
2013 \text { [39] }\end{array}$} & \multirow{5}{*}{$\begin{array}{l}\text { Deriving } \\
\text { network } \\
\text { properties to } \\
\text { describe the } \\
\text { network } \\
\text { (descriptive } \\
\text { SNA) }\end{array}$} & $\begin{array}{l}\mathrm{KT} \text { model } \\
\text { adopted }\end{array}$ & $\begin{array}{l}\text { Prestige within the } \\
\text { network }\end{array}$ & $\begin{array}{l}\text { Degree } \\
\text { centralization }\end{array}$ & \multirow{5}{*}{$\begin{array}{l}\text { KT teams with different models of KT (i.e., focus } \\
\text { on research dissemination vs. knowledge } \\
\text { co-production and brokering vs. integrated } \\
\text { research-clinical collaboration) } \\
\text { varied in their structural properties (e.g. the } \\
\text { prominence and control of leaders in KT } \\
\text { processes) }\end{array}$} \\
\hline & & & $\begin{array}{l}\text { Control over } \\
\text { knowledge }\end{array}$ & $\begin{array}{l}\text { Betweenness } \\
\text { centralization }\end{array}$ & \\
\hline & & & Access to knowledge & $\begin{array}{l}\text { Closeness } \\
\text { centralization }\end{array}$ & \\
\hline & & & $\begin{array}{l}\text { Alternate paths for } \\
\text { knowledge flow that } \\
\text { circumvent central } \\
\text { actors }\end{array}$ & $\begin{array}{l}\text { Flow betweenness } \\
\text { centralization }\end{array}$ & \\
\hline & & $\begin{array}{l}\text { Organizational } \\
\text { role (e.g., } \\
\text { director, support } \\
\text { staff) }\end{array}$ & $\begin{array}{l}\text { Core } \\
\text { actors-dominant } \\
\text { individuals with } \\
\text { frequent knowledge } \\
\text { exchange }\end{array}$ & $\begin{array}{l}\text { Coreness scores } \\
\text { (core-periphery } \\
\text { algorithm) }\end{array}$ & \\
\hline \multicolumn{6}{|c|}{ Predictive/explanatory studies } \\
\hline \multirow{4}{*}{$\begin{array}{l}\text { Zappa } 2011 \\
\text { [29] }\end{array}$} & \multirow{4}{*}{$\begin{array}{l}\text { Descriptive } \\
\text { SNA }\end{array}$} & \multirow{4}{*}{$\begin{array}{l}\text { External } \\
\text { communication } \\
\text { (\# visits from } \\
\text { drug } \\
\text { representatives); } \\
\text { research } \\
\text { orientation (\# } \\
\text { publications); } \\
\text { clinical } \\
\text { experience; } \\
\text { hierarchical } \\
\text { position } \\
\text { (administrative }\end{array}$} & \multirow{2}{*}{$\begin{array}{l}\text { Colleagues with } \\
\text { whom knowledge is } \\
\text { discussed and } \\
\text { transferred }\end{array}$} & Network density & Low network density (0.3\%) \\
\hline & & & & Components & \multirow{3}{*}{$\begin{array}{l}\text { Multiple small components suggested lack of } \\
\text { strong opinion leaders to drive treatment } \\
\text { adoption. Findings suggest physicians tend to } \\
\text { build small, closed, non-hierarchical internal, and } \\
\text { external connections within their professional } \\
\text { group, potentially limiting broader access to new } \\
\text { information } \\
\text { Isolates tended to be clinically experienced and } \\
\text { active in research. } \\
\text { Advice sharing more likely if physicians shared a } \\
\text { medical speciality, geographic proximity but }\end{array}$} \\
\hline & & & $\begin{array}{l}\text { Tendency to exchange } \\
\text { information with a } \\
\text { number of sources }\end{array}$ & Alternating k-stars & \\
\hline & & & $\begin{array}{l}\text { Tendency to share } \\
\text { knowledge within a } \\
\text { small peer group } \\
\text { (network closure) }\end{array}$ & $\begin{array}{l}\text { Alternating k- } \\
\text { triangles; alternat- } \\
\text { ing independent } \\
\text { two-paths }\end{array}$ & \\
\hline
\end{tabular}


Table 3 Variables, network properties and key findings (Continued)

\begin{tabular}{|c|c|c|c|c|c|}
\hline \multirow[t]{2}{*}{ Citation } & \multirow{2}{*}{$\begin{array}{l}\text { Primary data } \\
\text { analysis } \\
\text { method }\end{array}$} & \multicolumn{3}{|c|}{ Variables of interest } & \multirow[t]{2}{*}{ Findings } \\
\hline & & Attributes & $\begin{array}{l}\text { Structural or } \\
\text { relational parameters }\end{array}$ & $\begin{array}{l}\text { Network property } \\
\text { used as proxy for } \\
\text { structural } \\
\text { parameter }\end{array}$ & \\
\hline & & $\begin{array}{l}\text { role) } \\
\text { Medical } \\
\text { specialty* } \\
\text { Hospital } \\
\text { affiliation* }\end{array}$ & $\begin{array}{l}\text { Tendency to interact } \\
\text { with similar others } \\
\text { Prominence as a } \\
\text { knowledge source in } \\
\text { the network }\end{array}$ & $\begin{array}{l}\text { Tie homophily/ } \\
\text { hierarchy; } \\
\text { indegree centrality }\end{array}$ & $\begin{array}{l}\text { differed in research productivity or years in } \\
\text { practice. }\end{array}$ \\
\hline \multirow{12}{*}{$\begin{array}{l}\text { Yousefi- } \\
\text { Nooraie, } 2014 \\
\text { [37] (same } \\
\text { study as [36]) }\end{array}$} & \multirow[t]{4}{*}{$\begin{array}{l}\text { Descriptive } \\
\text { SNA }\end{array}$} & & $\begin{array}{l}\text { Relative } \\
\text { connectedness of } \\
\text { actors of a given role }\end{array}$ & $\begin{array}{l}\text { Indegree centrality } \\
\text { Outdegree } \\
\text { centrality }\end{array}$ & \multirow{12}{*}{$\begin{array}{l}\text { Managers identified as key brokers in KT } \\
\text { interventions and EIP implementation processes. } \\
\text { Public health professionals preferred to limit advice } \\
\text { seeking and expert recognition to a small number } \\
\text { of peers; advice seeking limited to own division. } \\
\text { Strong friendship ties a significant predictor of } \\
\text { information seeking ties. } \\
\text { EIP scores not predictive of information seeking or } \\
\text { expert recognition ties. }\end{array}$} \\
\hline & & $\begin{array}{l}\text { *Role (e.g., } \\
\text { manager) } \\
\text { *Organizational } \\
\text { division } \\
\text { Score on EIP } \\
\text { implementation } \\
\text { scale }\end{array}$ & Key individuals & Degree centrality & \\
\hline & & $\begin{array}{l}\text { Organizational } \\
\text { division }\end{array}$ & $\begin{array}{l}\text { Tendency to connect } \\
\text { to peers from other } \\
\text { units }\end{array}$ & E-l index & \\
\hline & & - & $\begin{array}{l}\text { Tendency to } \\
\text { reciprocate expert } \\
\text { recognition and } \\
\text { information seeking } \\
\text { ties }\end{array}$ & Tie reciprocity & \\
\hline & \multirow{5}{*}{$\begin{array}{l}\text { Exponential } \\
\text { random } \\
\text { graph } \\
\text { modeling } \\
\text { (ERGM) }\end{array}$} & \multirow{5}{*}{$\begin{array}{l}\text { *Role (e.g., } \\
\text { manager) } \\
\text { *Organizational } \\
\text { division } \\
\text { Score on EIP } \\
\text { implementation } \\
\text { scale }\end{array}$} & $\begin{array}{l}\text { Tendency to connect } \\
\text { with those with } \\
\text { similar attributes }\end{array}$ & Tie homophily & \\
\hline & & & Reciprocity & Tie reciprocity & \\
\hline & & & $\begin{array}{l}\text { Formation of } \\
\text { information seeking } \\
\text { and expertise- } \\
\text { recognition ties }\end{array}$ & $\begin{array}{l}\text { Ties and direction } \\
\text { of ties (in vs. out) }\end{array}$ & \\
\hline & & & $\begin{array}{l}\text { Tendency for } \\
\text { network to have } \\
\text { highly connected } \\
\text { nodes (hubs) }\end{array}$ & $\begin{array}{l}\text { Alternating in-k- } \\
\text { stars } \\
\text { Alternating out-k- } \\
\text { stars }\end{array}$ & \\
\hline & & & $\begin{array}{l}\text { Friendship } \\
\text { connections }\end{array}$ & Ties & \\
\hline & \multirow[t]{3}{*}{$\begin{array}{l}\text { Multilevel } \\
\text { logistic } \\
\text { regression }\end{array}$} & \multirow{3}{*}{$\begin{array}{l}\text { *Role (e.g., } \\
\text { manager) } \\
\text { *Organizational } \\
\text { division } \\
\text { Score on EIP } \\
\text { implementation } \\
\text { scale }\end{array}$} & $\begin{array}{l}\text { Tendency to connect } \\
\text { with those with } \\
\text { similar attributes }\end{array}$ & Tie homophily & \\
\hline & & & $\begin{array}{l}\text { Formation of } \\
\text { information seeking } \\
\text { and expertise- } \\
\text { recognition ties }\end{array}$ & Ties & \\
\hline & & & $\begin{array}{l}\text { Friendship } \\
\text { connections }\end{array}$ & Ties & \\
\hline $\begin{array}{l}\text { Tasselli, } 2015 \\
{[22]}\end{array}$ & $\begin{array}{l}\text { Paired } t \text { test } \\
\text { Linear } \\
\text { regression }\end{array}$ & $\begin{array}{l}{ }^{*} \text { Gender } \\
\text { *Tenure } \\
\text { *Profession } \\
\text { *Organizational } \\
\text { unit } \\
\text { *Rank (i.e., } \\
\text { leadership role) }\end{array}$ & $\begin{array}{l}\text { Ease of knowledge } \\
\text { transfer } \\
\text { Perceived receipt of } \\
\text { useful knowledge } \\
\text { Connectedness } \\
\text { Hierarchy } \\
\text { Network } \\
\text { fragmentation } \\
\text { Individual reach } \\
\text { Brokerage potential } \\
\text { *Network size }\end{array}$ & $\begin{array}{l}\text { Tie strength } \\
\text { Mean degree } \\
\text { centrality } \\
\text { Bonacich centrality } \\
\text { Mean } \\
\text { betweenness } \\
\text { centrality } \\
\text { Closeness } \\
\text { centrality } \\
\text { Betweenness } \\
\text { centrality }\end{array}$ & $\begin{array}{l}\text { Knowledge tends to transfer within rather than } \\
\text { across professions; nurses' networks were denser } \\
\text { and more hierarchical; closeness centrality } \\
\text { positively associated with ease of knowledge } \\
\text { transfer; brokering positions increased access to } \\
\text { useful knowledge }\end{array}$ \\
\hline
\end{tabular}


Table 3 Variables, network properties and key findings (Continued)

\begin{tabular}{|c|c|c|c|c|c|}
\hline \multirow[t]{2}{*}{ Citation } & \multirow{2}{*}{$\begin{array}{l}\text { Primary data } \\
\text { analysis } \\
\text { method }\end{array}$} & \multicolumn{3}{|c|}{ Variables of interest } & \multirow[t]{2}{*}{ Findings } \\
\hline & & Attributes & $\begin{array}{l}\text { Structural or } \\
\text { relational parameters }\end{array}$ & $\begin{array}{l}\text { Network property } \\
\text { used as proxy for } \\
\text { structural } \\
\text { parameter }\end{array}$ & \\
\hline & & & & *Degree centrality & \\
\hline $\begin{array}{l}\text { Menchik } 2010 \\
{[44]}\end{array}$ & $\begin{array}{l}\text { OLS } \\
\text { regression }\end{array}$ & $\begin{array}{l}\text { \# medical } \\
\text { literature } \\
\text { database } \\
\text { searches per } \\
\text { month } \\
\text { \# journals read } \\
\text { regularly } \\
\text { *Age } \\
\text { *Gender } \\
\text { *Tenure at } \\
\text { hospital } \\
\text { *Medical school } \\
\text { *\% clinical time } \\
\text { *Sub- } \\
\text { specialization } \\
\text { Prestige of } \\
\text { hospital } \\
\text { (published } \\
\text { rankings) }\end{array}$ & $\begin{array}{l}\text { Relational esteem by } \\
\text { colleagues }\end{array}$ & Indegree centrality & $\begin{array}{l}\text { Physicians in higher prestige hospitals were less } \\
\text { likely to be named as advice givers. Prestige in } \\
\text { these settings associated with medical school } \\
\text { attended. } \\
\text { In lower-prestige hospitals, regularly reading a } \\
\text { range of journals, and less time spent on clinical } \\
\text { work increased likelihood of high esteem by } \\
\text { colleagues }\end{array}$ \\
\hline $\begin{array}{l}\text { Mascia } 2014 \\
{[33]}\end{array}$ & $\begin{array}{l}\text { Ordinal } \\
\text { logistic } \\
\text { regression }\end{array}$ & $\begin{array}{l}\text { Self-reported } \\
\text { frequency of EIP } \\
\text { use } \\
\text { *Gender } \\
\text { *\# patients in } \\
\text { caseload } \\
\text { *area of clinical } \\
\text { practice (e.g., } \\
\text { asthma, urology, } \\
\text { etc.) } \\
\text { *\# article } \\
\text { subscriptions } \\
\text { *perceptions of } \\
\text { barriers to } \\
\text { availability of } \\
\text { evidence } \\
\text { *perceptions of } \\
\text { difficulty } \\
\text { applying } \\
\text { evidence to } \\
\text { practice } \\
\text { *Organizational } \\
\text { affiliation } \\
\text { *Affiliation to } \\
\text { formal groups } \\
\text { *Collaborative } \\
\text { nature of actor's } \\
\text { medical practice }\end{array}$ & $\begin{array}{l}\text { Degree of } \\
\text { collaboration with } \\
\text { colleagues }\end{array}$ & $\begin{array}{l}\text { Outdegree } \\
\text { centrality }\end{array}$ & $\begin{array}{l}\text { Degree centrality directly associated with } \\
\text { physicians' EIP use }\end{array}$ \\
\hline $\begin{array}{l}\text { Mascia } 2018 \\
{[27]}\end{array}$ & $\begin{array}{l}\text { Exponential } \\
\text { random } \\
\text { graph } \\
\text { models }\end{array}$ & $\begin{array}{l}\text { *Past task force } \\
\text { involvement } \\
{ }^{*} \text { Tenure } \\
{ }^{*} \text { Gender } \\
{ }^{*} \text { Geographic } \\
\text { distance } \\
{ }^{*} \text { Association } \\
\text { members } \\
\text { *Health district }\end{array}$ & $\begin{array}{l}\text { Tendency to } \\
\text { reciprocate advice } \\
\text { Tendency to seek } \\
\text { advice from an } \\
\text { indirect tie } \\
\text { Tendency for local, } \\
\text { generalized } \\
\text { exchange of advice } \\
\text { *Tendency to form } \\
\text { advice ties } \\
\text { *Popularity as an } \\
\text { advice source } \\
\text { *Advice-seeking } \\
\text { activity }\end{array}$ & $\begin{array}{l}\text { Tie reciprocity } \\
\text { Transitivity (path } \\
\text { closure) } \\
\text { Cyclic closure } \\
\text { *Density } \\
\text { *Indegree } \\
\text { centrality } \\
\text { *Outdegree } \\
\text { centrality } \\
\text { *Formation of } \\
\text { non-closure } \\
\text { structures } \\
\text { *Tie homophily (of } \\
\text { attributes) }\end{array}$ & $\begin{array}{l}\text { Advice ties unlikely unless reciprocated; advice ties } \\
\text { tended to organize around clusters-driven by } \\
\text { transitivity, not popularity; Tendency against } \\
\text { exchange of advice in cyclic structures; positive } \\
\text { relationship between ties and association } \\
\text { homophily in one health authority, and between } \\
\text { ties and district/task forces in the other; tendency } \\
\text { toward homophily related to tenure and distance, } \\
\text { but not gender }\end{array}$ \\
\hline
\end{tabular}


Table 3 Variables, network properties and key findings (Continued)

\begin{tabular}{|c|c|c|c|c|c|}
\hline \multirow[t]{2}{*}{ Citation } & \multirow{2}{*}{$\begin{array}{l}\text { Primary data } \\
\text { analysis } \\
\text { method }\end{array}$} & \multicolumn{3}{|l|}{ Variables of interest } & \multirow[t]{2}{*}{ Findings } \\
\hline & & Attributes & $\begin{array}{l}\text { Structural or } \\
\text { relational parameters }\end{array}$ & $\begin{array}{l}\text { Network property } \\
\text { used as proxy for } \\
\text { structural } \\
\text { parameter }\end{array}$ & \\
\hline & & & *Brokering & & \\
\hline $\begin{array}{l}\text { Mascia } 2015 \\
\text { [48] (same } \\
\text { data as [31], } \\
\text { [30] and [32]) }\end{array}$ & $\begin{array}{l}\text { Multiple } \\
\text { regression- } \\
\text { quadratic as- } \\
\text { signment } \\
\text { procedure } \\
\text { (MR-QAP) }\end{array}$ & $\begin{array}{l}\text { Age } \\
\text { Gender } \\
\text { Tenure/seniority }\end{array}$ & $\begin{array}{l}\text { Frequency of } \\
\text { collaboration } \\
\text { Similarity of } \\
\text { professional role, } \\
\text { institution and } \\
\text { geographical location }\end{array}$ & $\begin{array}{l}\text { Tie strength } \\
\text { Tie homophily }\end{array}$ & $\begin{array}{l}\text { Ties more likely if specialization, institution were } \\
\text { the same between individuals; less likely if similar } \\
\text { roles, greater difference in time since graduation } \\
\text { and further geographic distance; professional } \\
\text { homophily better predictor than institutional } \\
\text { homophily }\end{array}$ \\
\hline \multirow{3}{*}{$\begin{array}{l}\text { Mascia } 2013 \\
\text { [32] (same } \\
\text { data as [48], } \\
\text { [30] and [31]) }\end{array}$} & $\begin{array}{l}\text { Descriptive } \\
\text { SNA }\end{array}$ & \multirow{3}{*}{$\begin{array}{l}\text { Age* } \\
\text { Gender* } \\
\text { Hospital tenure* } \\
\text { Tenure in health } \\
\text { authority* } \\
\text { Managerial role* } \\
\text { Geographical } \\
\text { distance from } \\
\text { colleagues* } \\
\text { Affiliation with } \\
\text { other } \\
\text { organizations* } \\
\text { Self-reported ElP } \\
\text { adoption (i.e. } \\
\text { frequency of } \\
\text { database } \\
\text { searching) }\end{array}$} & Connectedness & $\begin{array}{l}\text { Whole network } \\
\text { density }\end{array}$ & Low density (5.7\%) observed \\
\hline & \multirow[t]{2}{*}{$\begin{array}{l}\text { OLS } \\
\text { regression }\end{array}$} & & $\begin{array}{l}\text { Network authority (i.e., } \\
\text { importance—relevant } \\
\text { and popular) }\end{array}$ & $\begin{array}{l}\text { Hubs and } \\
\text { authorities } \\
\text { centrality }\end{array}$ & \multirow[t]{2}{*}{$\begin{array}{l}\text { The most active EIP practitioners likely to be found } \\
\text { at network periphery (i.e. least central) }\end{array}$} \\
\hline & & & $\begin{array}{l}\text { Degree of coreness in } \\
\text { the network }\end{array}$ & $\begin{array}{l}\text { Network coreness } \\
\text { score (degree } \\
\text { centrality and } \\
\text { core-periphery } \\
\text { position) }\end{array}$ & \\
\hline \multirow[t]{2}{*}{$\begin{array}{l}\text { Mascia 2011b } \\
\text { [31] (same } \\
\text { data as [48], } \\
\text { [32] and [30]) }\end{array}$} & \multirow[t]{2}{*}{$\begin{array}{l}\text { Ordinal } \\
\text { logistic } \\
\text { regression }\end{array}$} & \multirow{2}{*}{$\begin{array}{l}\text { Tendency to } \\
\text { adopt EIP (self- } \\
\text { reported } \\
\text { frequency of peer- } \\
\text { reviewed research } \\
\text { use) } \\
\text { Age* } \\
\text { Gender* } \\
\text { Tenure in health } \\
\text { authority/ } \\
\text { organization* } \\
\text { Managerial role* } \\
\text { \# publications* } \\
\text { Perceived access } \\
\text { to evidence* } \\
\text { Hospital } \\
\text { affiliation* }\end{array}$} & $\begin{array}{l}\text { Extent to which a } \\
\text { given tie is } \\
\text { redundant because } \\
\text { of concurrent ties } \\
\text { with another alter }\end{array}$ & $\begin{array}{l}\text { Ego-network } \\
\text { constraint }\end{array}$ & \multirow{2}{*}{$\begin{array}{l}\text { Physicians with greater network constraint (i.e., } \\
\text { many redundant ties) reported decreased EIP } \\
\text { adoption. May be related to information } \\
\text { bias-tendency of physicians to interpret the } \\
\text { information in a way that is congruent with their } \\
\text { previous knowledge or opinion. } \\
\text { High degree centrality associated with EIP use. }\end{array}$} \\
\hline & & & $\begin{array}{l}\text { Individual's network } \\
\text { size* }^{*}\end{array}$ & $\begin{array}{l}\text { Total \# of ties in } \\
\text { ego-network } \\
\text { (indegree }+ \text { outde- } \\
\text { gree centrality)* }\end{array}$ & \\
\hline \multirow{4}{*}{$\begin{array}{l}\text { Mascia 2011a } \\
\text { [30] (same } \\
\text { data as [32, } \\
48] \text { ) }\end{array}$} & \multirow[t]{2}{*}{$\begin{array}{l}\text { Descriptive } \\
\text { SNA }\end{array}$} & \multirow[t]{2}{*}{-} & $\begin{array}{l}\text { Average number of } \\
\text { advice exchange } \\
\text { colleagues }\end{array}$ & $\begin{array}{l}\text { Mean ego-network } \\
\text { density }\end{array}$ & \multirow{4}{*}{$\begin{array}{l}\text { Advice sharing most likely when physicians shared } \\
\text { a medical specialty, geographic proximity, similar } \\
\text { attitudes toward EIP, or had co-authored } \\
\text { publications. } \\
\text { Collaboration less likely when actors held similar } \\
\text { managerial roles, or were at different hospitals/ } \\
\text { clinical/geographical areas. }\end{array}$} \\
\hline & & & $\begin{array}{l}\text { Tendency for } \\
\text { colleagues to both } \\
\text { give and receive } \\
\text { advice with one } \\
\text { another }\end{array}$ & Tie reciprocity & \\
\hline & \multirow{2}{*}{$\begin{array}{l}\text { Multiple } \\
\text { regression } \\
\text { quadratic } \\
\text { assignment } \\
\text { procedures } \\
\text { (MR-QAP } \\
\text { analysis) }\end{array}$} & \multirow[t]{2}{*}{-} & $\begin{array}{l}\text { Advice exchange } \\
\text { among pairs of } \\
\text { physicians }\end{array}$ & Ego-network ties & \\
\hline & & & $\begin{array}{l}\text { Similarity between } \\
\text { pairs of tied actors in: } \\
\text { Geographical } \\
\text { distances } \\
\text { Gender* } \\
\text { Age* }^{*} \\
\text { Medical } \\
\text { specialization* }\end{array}$ & Tie homophily & \\
\hline
\end{tabular}


Table 3 Variables, network properties and key findings (Continued)

\begin{tabular}{|c|c|c|c|c|c|}
\hline \multirow[t]{2}{*}{ Citation } & \multirow{2}{*}{$\begin{array}{l}\text { Primary data } \\
\text { analysis } \\
\text { method }\end{array}$} & \multicolumn{3}{|l|}{ Variables of interest } & \multirow[t]{2}{*}{ Findings } \\
\hline & & Attributes & $\begin{array}{l}\text { Structural or } \\
\text { relational parameters }\end{array}$ & $\begin{array}{l}\text { Network property } \\
\text { used as proxy for } \\
\text { structural } \\
\text { parameter }\end{array}$ & \\
\hline \multirow{3}{*}{ Paul 2015 [35] } & \multirow{3}{*}{$\begin{array}{l}\text { Extended } p_{2} \\
\text { model with } \\
\text { Bayesian } \\
\text { modeling } \\
\text { and } \\
\text { estimation }\end{array}$} & & $\begin{array}{l}\text { Clinical experience* } \\
\text { Tenure in health } \\
\text { authority/ } \\
\text { organization* } \\
\text { Managerial role* } \\
\text { \# publications* } \\
\text { Co-authorship* } \\
\text { ElP adoption (self- } \\
\text { reported frequency } \\
\text { of peer-reviewed re- } \\
\text { search use)* }\end{array}$ & & \\
\hline & & $\begin{array}{l}\text { *Age } \\
\text { Patient age } \\
\text { Patient sex } \\
\text { Patient race } \\
\text { Patient health } \\
\text { status } \\
\text { Patient intensity } \\
\text { of care }\end{array}$ & $\begin{array}{l}\text { Relative \# shared } \\
\text { patients } \\
\text { *Same gender } \\
\text { *Same specialty } \\
\text { *Same location } \\
\text { Reciprocity } \\
\text { Social dependence } \\
\text { (clustering) }\end{array}$ & $\begin{array}{l}\text { Density } \\
\text { Tie homophily } \\
\text { Tie reciprocity } \\
\text { Alternating } \\
\text { independent two- } \\
\text { paths } \\
\text { Transitive triads } \\
\text { Alternating k-stars } \\
\text { (two-stars) }\end{array}$ & $\begin{array}{l}\text { Low network density }(0.10) \text { observed. } \\
\text { Triadic clustering higher than chance. } \\
\text { Ties not associated with gender/specialty } \\
\text { homophily. } \\
\text { Location positively associated with ties. } \\
\text { Complementary expertise positively associated } \\
\text { with patient sharing. } \\
\text { Transitivity may account for reciprocity }\end{array}$ \\
\hline & & \multirow{4}{*}{$\begin{array}{l}\text { Gender } \\
\text { Clinic } \\
\% \text { female } \\
\text { patients } \\
\text { Self-identify as } \\
\text { expert } \\
\text { \# clinics per } \\
\text { week } \\
\text { \# years practicing } \\
\text { in the city } \\
\text { Tenure at } \\
\text { hospital } \\
\text { Years clinical } \\
\text { experience } \\
\text { Location of } \\
\text { training }\end{array}$} & $\begin{array}{l}\text { Involvement in } \\
\text { influential discussions }\end{array}$ & $\begin{array}{l}\text { Whole network } \\
\text { density }\end{array}$ & $\begin{array}{l}\text { Low density (0.154) observed; reciprocity more } \\
\text { likely than not—-may be an artifact of transitivity; } \\
\text { high triadic clustering observed; same clinic and } \\
\text { gender, expert, higher clinical caseload increased } \\
\text { tendency for tie formation }\end{array}$ \\
\hline \multirow[t]{3}{*}{$\begin{array}{l}\text { Keating } 2007 \\
{[45]}\end{array}$} & \multirow{3}{*}{$\begin{array}{l}\text { P2 logistic } \\
\text { regression } \\
\text { analysis }\end{array}$} & & $\begin{array}{l}\text { Being perceived as } \\
\text { influential }\end{array}$ & Indegree centrality & \multirow{3}{*}{$\begin{array}{l}\text { Self-identified experts seen as more influential; no } \\
\text { relationship between \# years in practice or location } \\
\text { of work or training. } \\
\text { Clustering with respect to EIP knowledge } \\
\text { exchange observed between those with greater \# } \\
\text { of patients and higher frequency of clinical } \\
\text { sessions. } \\
\text { High reciprocity observed in the absence of } \\
\text { opinion leaders with high centrality. }\end{array}$} \\
\hline & & & $\begin{array}{l}\text { Perceiving others as } \\
\text { influential } \\
\text { (information seeking) }\end{array}$ & $\begin{array}{l}\text { Outdegree } \\
\text { centrality }\end{array}$ & \\
\hline & & & Reciprocity & Tie reciprocity & \\
\hline $\begin{array}{l}\text { Heijmans } 2017 \\
{[26]}\end{array}$ & $\begin{array}{l}\text { Paired } \\
\text { sample } t \\
\text { tests/ } \\
\text { Wilcoxon } \\
\text { tests } \\
\text { Logistic } \\
\text { multi-level } \\
\text { analyses }\end{array}$ & $\begin{array}{l}\text { *Patient age } \\
\text { *Patient sex } \\
\text { *Patient group } \\
\text { *Patient illness } \\
\text { status } \\
\text { *Treatment/ } \\
\text { control group for } \\
\text { parallel } \\
\text { randomized } \\
\text { controlled trial }\end{array}$ & $\begin{array}{l}\text { Connectedness } \\
\text { Frequency of contact } \\
\text { Influence of } \\
\text { coordinator } \\
\text { Similarity in attitudes } \\
\text { related to treatment } \\
\text { goals } \\
\text { Presence of opinion } \\
\text { leader } \\
\text { *Network size }\end{array}$ & $\begin{array}{l}\text { Density } \\
\text { Tie strength } \\
\text { Degree centrality } \\
\text { Homophily (E-I } \\
\text { index) } \\
\% \text { of possible in- } \\
\text { degree ties }\end{array}$ & $\begin{array}{l}\text { Low density ( } 0.37 \text { and } 0.38 \text { ) observed. } \\
\text { Most ties between those who did not value } \\
\text { achieving treatment goals. } \\
\text { General practitioner most likely named as opinion } \\
\text { leader. } \\
\text { Nurse performance associated with consistently } \\
\text { identified opinion leader. } \\
\text { Lack of tie homophily for positive attitudes } \\
\text { associated with poor clinical outcomes }\end{array}$ \\
\hline $\begin{array}{l}\text { Friedkin } 2010 \\
{[25]}\end{array}$ & $\begin{array}{l}\text { Random } \\
\text { intercept } \\
\text { multi-level } \\
\text { event history } \\
\text { model }\end{array}$ & $\begin{array}{l}\text { Professional age } \\
\text { Chief or honorary } \\
\text { position (yes/no) } \\
\text { Number of } \\
\text { journals read } \\
\text { Value keeping up } \\
\text { with scientific } \\
\text { developments } \\
\text { Physicians' } \\
\text { adoption of a } \\
\text { new antibiotic } \\
\text { (i.e., prescribing } \\
\text { behavior) } \\
\text { Marketing } \\
\text { patterns of drug } \\
\text { companies }\end{array}$ & $\begin{array}{l}\text { Influence of advisors/ } \\
\text { discussion partners }\end{array}$ & $\begin{array}{l}\text { Contact network } \\
\text { role (CNET)_a } \\
\text { summative } \\
\text { measure of } 4 \\
\text { measures of } \\
\text { structural cohesion } \\
\text { and structural } \\
\text { equivalence; } \\
\text { position in the } \\
\text { medical advice } \\
\text { network }\end{array}$ & $\begin{array}{l}\text { Cohesion and structural equivalence were } \\
\text { correlated, and may be useful in combination to } \\
\text { improve reliability in the evaluation of network } \\
\text { structures across settings }\end{array}$ \\
\hline
\end{tabular}


Table 3 Variables, network properties and key findings (Continued)

\begin{tabular}{|c|c|c|c|c|c|}
\hline \multirow[t]{2}{*}{ Citation } & \multirow{2}{*}{$\begin{array}{l}\text { Primary data } \\
\text { analysis } \\
\text { method }\end{array}$} & \multicolumn{3}{|l|}{ Variables of interest } & \multirow[t]{2}{*}{ Findings } \\
\hline & & Attributes & $\begin{array}{l}\text { Structural or } \\
\text { relational parameters }\end{array}$ & $\begin{array}{l}\text { Network property } \\
\text { used as proxy for } \\
\text { structural } \\
\text { parameter }\end{array}$ & \\
\hline & & $\begin{array}{l}\text { Proportion of } \\
\text { previous } \\
\text { adopters at a } \\
\text { given time } \\
\text { ("internal } \\
\text { contagion") }\end{array}$ & & & \\
\hline $\begin{array}{l}\text { Di Vincenzo } \\
2017 \text { [28] }\end{array}$ & $\begin{array}{l}\text { Ordinary } \\
\text { Least } \\
\text { Squares } \\
\text { regression }\end{array}$ & $\begin{array}{l}\text { \# publications } \\
\text { *Tenure } \\
\text { *Managerial role } \\
\text { *Geographic } \\
\text { distance } \\
\text { *Hospital } \\
\text { affiliation } \\
\text { *\# publications } \\
\text { from same- } \\
\text { specialty } \\
\text { colleagues }\end{array}$ & $\begin{array}{l}\text { Dependence on } \\
\text { others/access to new } \\
\text { information } \\
\text { Relative productivity } \\
\text { among ego-network } \\
\text { colleagues } \\
\text { Same role } \\
\text { Same specialty }\end{array}$ & $\begin{array}{l}\text { Ego-network } \\
\text { constraint } \\
\text { Euclidian distance } \\
\text { *Ego-network size }\end{array}$ & $\begin{array}{l}\text { Young employees appeared to have more } \\
\text { redundant networks (greater need for advice). } \\
\text { Hospital affiliation (i.e., context) influenced } \\
\text { constraint. } \\
\text { Constraint negatively associated with ego-network } \\
\text { size and relative productivity (mediated by profes- } \\
\text { sional group membership), positively with product- } \\
\text { ivity, Euclidean distance, role/specialty homophily } \\
\text { (augments impact of productivity on prestige) }\end{array}$ \\
\hline \multirow[t]{2}{*}{ Burt 1987 [24] } & \multirow{2}{*}{$\begin{array}{l}\text { Ordinary } \\
\text { least squares } \\
\text { regression } \\
\text { with } \\
\text { likelihood- } \\
\text { ratio chi- } \\
\text { squared test }\end{array}$} & \multirow{2}{*}{$\begin{array}{l}\text { Timing of } \\
\text { adoption } \\
\text { Relative timing of } \\
\text { adoption within } \\
\text { the network } \\
\text { Professional age } \\
\text { Contact with } \\
\text { drug company } \\
\text { Number of } \\
\text { journals read } \\
\text { Number of } \\
\text { house calls vs. } \\
\text { office visits } \\
\text { Value keeping up } \\
\text { with scientific } \\
\text { developments }\end{array}$} & $\begin{array}{l}\text { Position in the } \\
\text { medical advice/ } \\
\text { discussion network }\end{array}$ & $\begin{array}{l}\text { Structural } \\
\text { equivalence }\end{array}$ & \multirow{2}{*}{$\begin{array}{l}\text { Adoption by others in equivalent positions within } \\
\text { the network was a stronger predictor of adoption } \\
\text { than adoption by those in an individual's advice or } \\
\text { discussion networks. } \\
\text { Early adopters tended to participate in a range of } \\
\text { EIP behaviors. } \\
\text { Adoption by prominent physicians seen to be } \\
\text { related to their desire to avoid being late adopters. }\end{array}$} \\
\hline & & & $\begin{array}{l}\text { Influence of advisors/ } \\
\text { discussion partners }\end{array}$ & $\begin{array}{l}\text { Structural } \\
\text { cohesion }\end{array}$ & \\
\hline \multirow[t]{3}{*}{$\begin{array}{l}\text { Ankem } 2003 \\
\text { [23] }\end{array}$} & $\begin{array}{l}\text { Chi-square } \\
\text { statistics }\end{array}$ & $\begin{array}{l}\text { Preferred } \\
\text { information } \\
\text { source } \\
\text { Timing of } \\
\text { awareness of the } \\
\text { intervention } \\
\text { Timing of } \\
\text { intervention } \\
\text { adoption }\end{array}$ & - & - & $\begin{array}{l}\text { Clinical networks were most prominent in fostering } \\
\text { awareness and adoption of a clinical intervention, } \\
\text { but research and social networks also likely to } \\
\text { influence these processes. } \\
\text { Early adopters tended to rely on journals and } \\
\text { conferences for information informing practice } \\
\text { change; late adopters to a greater extent by } \\
\text { network contacts. }\end{array}$ \\
\hline & \multirow[t]{2}{*}{$\begin{array}{l}\text { Factor } \\
\text { analysis }\end{array}$} & \multirow{2}{*}{$\begin{array}{l}\text { Specialization } \\
\text { Hospital } \\
\text { City } \\
\text { Timing of } \\
\text { adoption } \\
\text { Frequency of } \\
\text { communication } \\
\text { with colleagues }\end{array}$} & $\begin{array}{l}\text { Types of relations } \\
\text { within the network } \\
\text { (e.g., clinical, research, } \\
\text { leisure) }\end{array}$ & Ties & \\
\hline & & & $\begin{array}{l}\text { Groupings of } \\
\text { information exchange } \\
\text { relations }\end{array}$ & Cliques & \\
\hline \multicolumn{6}{|c|}{ Longitudinal evaluative studies } \\
\hline $\begin{array}{l}\text { Racko } 2018 \\
{[47]}\end{array}$ & $\begin{array}{l}\text { Ordinary } \\
\text { least squares } \\
\text { regression }\end{array}$ & $\begin{array}{l}\text { Professional } \\
\text { status (ranking) } \\
\text { *Professional role } \\
{ }^{*} \text { Gender } \\
\text { *Education } \\
{ }^{*} \text { Organizational } \\
\text { status }\end{array}$ & $\begin{array}{l}\text { Research } \\
\text { collaboration } \\
\text { Joint decision- } \\
\text { making } \\
\text { Connectedness to } \\
\text { high-status } \\
\text { individuals } \\
\text { Connectedness to } \\
\text { knowledge brokers }\end{array}$ & $\begin{array}{l}\text { Ego-network size } \\
\text { via tie heterophily } \\
\text { Tie strength } \\
\text { Mean status score } \\
\text { of ego-network } \\
\text { relative to whole } \\
\text { network } \\
\% \text { of possible ties } \\
\text { *Tie homophily }\end{array}$ & $\begin{array}{l}\text { Higher social status associated with more research } \\
\text { collaboration at all time points, and joint decision- } \\
\text { making in early phases. } \\
\text { Higher-status ties with peers, ties to formal } \\
\text { knowledge brokers and ties to unfamiliar peers } \\
\text { inconsistently predicted knowledge exchange, } \\
\text { research collaboration and joint decision-making } \\
\text { over time. } \\
\text { Formal knowledge broker presence may facilitate }\end{array}$ \\
\hline
\end{tabular}


Table 3 Variables, network properties and key findings (Continued)

\begin{tabular}{|c|c|c|c|c|c|}
\hline \multirow[t]{2}{*}{ Citation } & \multirow{2}{*}{$\begin{array}{l}\text { Primary data } \\
\text { analysis } \\
\text { method }\end{array}$} & \multicolumn{3}{|c|}{ Variables of interest } & \multirow[t]{2}{*}{ Findings } \\
\hline & & Attributes & $\begin{array}{l}\text { Structural or } \\
\text { relational parameters }\end{array}$ & $\begin{array}{l}\text { Network property } \\
\text { used as proxy for } \\
\text { structural } \\
\text { parameter }\end{array}$ & \\
\hline & & & $\begin{array}{l}\text { Connectedness to } \\
\text { unfamiliar peers } \\
\text { *Intra-professional } \\
\text { whole network size }\end{array}$ & & interprofessional networking \\
\hline $\begin{array}{l}\text { Bunger } 2016 \\
\text { [43] }\end{array}$ & $\begin{array}{l}\text { Paired } t \text { tests; } \\
\text { one-way } \\
\text { analysis of } \\
\text { variance; de- } \\
\text { scriptive SNA }\end{array}$ & $\begin{array}{l}\text { Role (faculty } \\
\text { expert, internal } \\
\text { colleague, } \\
\text { external peer, } \\
\text { private } \\
\text { practitioner, } \\
\text { other) }\end{array}$ & $\begin{array}{l}\text { Connectedness } \\
\text { Lack of advice } \\
\text { seeking/sharing } \\
\text { Reciprocity } \\
\text { Similarity in } \\
\text { connectedness } \\
\text { Tendency for sub- } \\
\text { groups to form } \\
\text { Same institution } \\
\text { Frequency of } \\
\text { communication }\end{array}$ & $\begin{array}{l}\text { Density } \\
\text { Isolates } \\
\text { Tie reciprocity } \\
\text { Indegree } \\
\text { centralization } \\
\text { Clustering } \\
\text { Tie homophily } \\
\text { Tie strength }\end{array}$ & $\begin{array}{l}\text { Ego-network size decreased, more markedly for } \\
\text { senior leaders. } \\
\text { Exposure to private practitioners and "others" } \\
\text { decreased; exposure to experts increased. } \\
\text { Substantial turnover in dyads was reported, with } \\
\text { greater tie density around central core of experts. } \\
\text { Reciprocity and tie heterophily increased over time }\end{array}$ \\
\hline
\end{tabular}

$K T$ knowledge translation, EIP evidence informed practice. Where indicated by the article's author, italic text $=$ dependent variable; ${ }^{*}=$ covariate $^{\prime}$

sharing the same profession with a connection) would be associated with greater knowledge transfer ease, the presence of brokers (bridgers) would be associated with an increase in the receipt of useful information, particularly to managers, and that greater connectivity, frequency of contact, homophily, the presence of a highly connected clinical coordinator, and being an opinion leader would be associated with an increase the use of best practices. For more information about the full array of correlational, dependent and independent variables and covariates (both relational variables and attributes) identified in the included studies, refer to Table 3.

Information seeking patterns varied across professions and networks, with health professionals from some disciplines having a tendency to form small, closed subgroups, while others demonstrated greater connectivity and reach within the network, increased hierarchy (e.g., reliance on "gatekeepers" of information spreading it in a top-down approach), or relied more on sources of information or support external to the network (e.g., other organizations). Available information suggests that isolated individuals and those less connected at the periphery of the network may have more clinical experience and be more evidence-based in their practices than those at the network core $[29,32]$. In the absence of a core-periphery structure (i.e., a more highly connected center with a less connected network periphery), degree centrality (i.e., the number of connections an individual has) may be a key factor associated with EIP use, at least for physicians [33]. A network-identified broker or opinion leader may increase access to useful knowledge [14], improve practice performance [26], and facilitate networking across professions [47].

\section{Network properties}

Table 3 summarizes the key variables under study, the network properties that were derived from relational data, and the relational parameters (i.e., the constructs for which the network properties were acting as proxies). For example, network density was used as a proxy for connectedness in one study, and for representing the number of shared patients in another. Attributes of interest (which included individual characteristics, such as profession and gender; environmental characteristics, such as organization; social attributes, such as perceived reputation; and KT-related measures, such as EIP attitude scores) are also presented to offer a summary of the nature of non-SNA variables that have been analyzed alongside network properties. Study findings are presented in the final column for interest.

Eleven articles explored only a single or pair of network properties. Although 28 network properties were identified during data extraction, the majority of authors examined centrality, tie characteristics (e.g., the directions of the interactions; similarity in characteristics among pairs of connected individuals), and density (i.e., the proportion of ties relative to all possible ties) as their network properties of interest. Tie homophily (i.e., similarity of connected individuals on a given attribute, such as gender), indegree centrality (i.e., the number of people naming an individual as being connected to them), whole network density, the presence of ties, and tie reciprocity (i.e., bi-directionality in reported interactions or connections) were the most prevalent structural properties studied. The study authors' discussions about the influences of these network properties were clearly linked to prominent theoretical 
perspectives. Less emphasis was placed on the analysis of centralization (i.e., the evenness of the distribution of connections), subgroups (i.e., groups of connected individuals not connected to other groups within the network), and transitivity (i.e., patterns related to sets of three individuals and their tendencies to share connections with one another).

Attributes, such as research versus clinical productivity, professional field or specialty, leadership role and organizational prestige $[29,30,35,38,44,48]$, as well as the presence of other types of ties (e.g., friendship, expertise recognition, previous collaborations) $[30,36]$, appear to be predictive or explanatory factors for the formation of information seeking or research collaboration ties. Conflicting findings regarding the influence of EIP attitudes, experience, gender, and geographical proximity on tie formation were identified [27, 30, 35, 37, 45].

\section{Use of theory}

Diffusion of innovation was the theory most frequently applied (seven articles) $[23-25,29,33,34$, $38]$; social contagion theory [24, 25,33] and social influence theory $[33,36,44]$ were used in four and three studies, respectively. A model combining transactive memory theory and social exchange theory [37], as well as Habermas' theory of communicative power [42], social capital theory [47], sociology of professions theory [22], balance theory [27], and an epistemic differences perspective [39], were applied in one instance each. Most commonly, theory was used to select network properties to examine and to develop hypotheses to test. In addition, theory was used to provide background information about SNA or the topic under study, to assist in the interpretation of findings, and to develop and test new analytical methods to advance the field of SNA.

Several other articles employed SNA-specific theoretical perspectives to exploratory analysis, including applying Granovetter's strength of weak ties perspective [33], examining the association between structural holes (i.e., areas lacking connections) and the establishment of brokers that bridge network gaps [27, 28] or between a lack of ties and EIP attitudes [32], examining the role of social pressure on tie formation $[24,25]$, exploring the influence of being within a highly connected core of the network versus a less connected peripheral area on attitudes toward EIP [32], and evaluating network dynamics relative to the homophily principle (i.e., the tendency of people to form connections with similar others) [27, 28]. Seven articles employed a SNA paradigm without reference to a specific theory $[21,26,35,40,41,43,45]$.

\section{Discussion}

\section{Publication characteristics}

While SNA has a long history in fields, such as sociology, mathematics, and psychology, its popularity in health care has shown momentum since the early 2000s [6]. Annual publication trends suggest an emerging interest in SNA as an approach to study KT processes and determinants since 2010. This concentration follows work by Thomas Valente and colleagues in the mid-late 2000s. Their work applied SNA in health care to identify and to evaluate brokers and opinion leaders as a means of promoting behavior change, linked communication networks and diffusion principles to health promotion research, and applied network principles to the study of community-based cancer research [49-51]. Increasing interest in the utility of SNA across a range of health care contexts, and the relative maturity of this field, may contribute to its continued use in the newer field of KT to embed new practices across settings.

\section{Study design and data collection}

More longitudinal research would allow us to determine the direction of the causal relationships between network structures and attribute variables, such as EIP attitudes and behaviors, for better prediction of implementation outcomes. Such research would also enable the evaluation of changes in network connection patterns over time. This approach can be used to assess the impact of network interventions. Network interventions can use socially based strategies to identify and target network gaps [52] (e.g., interactive forums to help establish connections for isolated individuals or groups) to enhance KT processes, organizational capacity, or adherence to desired behavior (e.g., through social influence) [53]. Network interventions may also harness strengths in a network $[52,53]$ (e.g., engaging highly connected individuals to exert influence or to share resources) to better mobilize EIP attitudes, behaviors, or information flow for sustained implementation.

The simulation investigation for empirical network analysis (SIENA) framework offers a means of evaluating whole network dynamics, particularly with networks defined by connections that persist over time, which could be particularly helpful in identifying barriers to the successful introduction of interventions in specific settings [54]. The SIENA framework can evaluate change in repeated measures of a network with respect to actors and ties, as well as the interplay of these network changes alongside changes in the behaviour of network members [54]. For example, a network intervention aimed at reducing isolation within the network, or at increasing the connectedness of individuals who are positioned well to influence many others, can be evaluated for its 
effectiveness using the SIENA framework. The evidence of these changes would serve as supporting mechanisms for new evidence-based interventions. Computational models, such as agent-based modeling, can also be used to represent individuals and their interactions. Multiple simulation experiments that are programmed based on attribute data and structural characteristics allow researchers to specify and to control the parameters of the computational algorithms in order to determine the effects of specific variables [55].

Survey use was the dominant method of SNA data collection employed, which is consistent with the broader SNA field [7]. Although interviews were not prominent in the reviewed articles, they can gather the same relational data as surveys, while allowing the participant and interviewer to clarify question and response meaning (e.g., defining operational terms in more depth; describing the reasoning behind naming specific people in one's network) [56]. A qualitative data collection approach may have implications for the size of the sample, but the opportunity for discussion can increase response validity, while the relational network data can be quantified for analysis [56]. Qualitative interpretive strategies can also be used to understand context and meaning within the network and the phenomenon of interest [56], such as the extent to which network structure and/or specific attributes or contextual factors are perceived to influence attitudes, knowledge, or behavior related to the introduction of a new intervention. Mixed methods can be used to triangulate findings to validate results, and to strengthen the explanatory power of the research by exploring the complexities involved to a greater depth [56].

Limited use of document review was also observed, none of which involved electronic data. The use of secondary data (e.g., email records, social media interactions, medical records) to quantify networks may be more or less time intensive than primary data collection; limitations may also exist in the types of variables available to study [7]. However, particularly with electronic data, documentation of network activity may be readily available through regular quality monitoring, and span a time period to enable longitudinal analysis [7]. For example, data related to evidence sharing communication patterns and subsequent use of best practices by health professionals (e.g., intervention approaches, treatment intensity, or dosage) within and across clinical teams can be leveraged to identify network strengths and gaps, and to monitor KT strategy effectiveness.

Observation is a fourth means of SNA data collection, which was absent from the reviewed studies. Although more resource-dependent and not without risk of observer-influenced behavior change, observation may enable the identification of ties not captured through self-report [7]. For example, interpersonal dynamics during a meeting may be recorded by a third party more objectively than meeting participants may recall, while concurrently focusing on the content of the meeting. Self-report data also presents potential bias related to recall, particularly when respondents are asked to think back to interactions in the past, or to report their frequencies [7]. While network rosters can be used to help mitigate this problem in clearly bounded networks (e.g., an organization), in larger networks this strategy can create excessive burden on respondents [7]. Careful attention to the way questions are worded, and consideration of the number of alters requested of respondents must be made to gather meaningful and accurate data [7].

\section{Networks and actors}

With more than half of the included studies examining physician-only networks, and only a handful studying interprofessional health care teams, great opportunity exists to expand the range of professions under study. Because of the growing shift in health services delivery from professionbased to collaborative practice models involving interprofessional teams [57], further research is needed to evaluate the generalizability of findings beyond physician networks, as well as in other health care contexts. The diversity of network sizes and settings, however, demonstrates the utility of SNA for a broad range of applications, from the study of interventions in small hospital health care teams to large multi-organizational or national networks. The examination of inter-organizational networks (i.e., organizations as nodes) in the context of $\mathrm{KT}$ was beyond the scope of this review. Further study may be warranted to scope out this literature and to determine its implications for informing KT from an organizational network perspective.

\section{Study purposes and data analysis methods}

The examination of information exchange processes, key players, reasons for tie patterns, associations between network properties and various attributes, and the evaluation of KT intervention outcomes are crucial for understanding how to successfully embed a new practice. However, because of its examination of information flow, predominantly, this body of research presents a narrow view of KT that focuses primarily at the individual level of evidence-based decision-making. This limitation relates in part to the scope of the review, as well as the consideration that other actors (e.g., health leaders, researchers) rather than health professionals may typically manage many of the KT activities that were not represented. Extending the application of SNA to broader organizational or group processes and through a wider range of KT-related activities and actors will advance our understanding of the network dynamics involved in 
all aspects required to move evidence into action. Such phenomena of interest may include the collaborative production of KT tools, barriers assessments, implementation processes, and evaluation efforts. Examining these processes from a network perspective has the potential to identify strengths and gaps in the network that need to be addressed, to explore the structural characteristics and associated attribute-level variables that might contribute to their success, to describe the role of health professionals in these processes, and to evaluate network-level KT interventions to facilitate them.

The limited number of network properties (i.e., three or fewer) examined in more than half of this body of literature suggests that the potential for greater SNA -related insights from these studies to inform future research and practice in KT remains largely untapped. Simply describing networks or examining a single network property (e.g., tie homophily, centrality) and its association with attribute variables fails to leverage SNA's full potential. As KT scientists, we are interested in not only what is happening, but why it occurs, and the processes involved. With this information, we are positioned more effectively to design network-based KT interventions.

For example, basic SNA can be used to identify key players with influence within the network; subsequent analyses can be used to explain how these individuals came to hold these positions. Knowledge of an individual's structural position may also help to determine from whom they may seek evidence or KT support. This information can be used to develop KT interventions that target specific health professionals or groups of individuals based on their network structure or key attributes to strengthen KT processes. For instance, influential individuals can be leveraged as champions or knowledge brokers to improve the efficiency of information exchange or behavioral influence within a discipline group. Individuals with attributes in common with key players can be selected to lead KT interventions within an interprofessional health care team. Alternate paths for efficient information exchange or behavioral influence can be accessed if resistance by specific individuals is encountered.

An understanding of relational influences can also advance the science of KT by improving the specificity of KT interventions, and by supporting their evaluation. For instance, KT intervention fidelity (e.g., intended versus actual information flow) can be monitored using SNA, and the KT intervention can be adjusted accordingly over time to address gaps or barriers. Network-specific outcomes of a KT intervention (e.g., increased connectedness, access to information) can also be evaluated empirically based on relational data. Collaboration between $\mathrm{KT}$ and SNA researchers may enable a more in-depth examination of the data available from KT research, to bring new insights from a network perspective.

Visualizations are an asset in SNA research because of their ability to represent the data in a way that makes it more accessible to those less familiar with SNA methodologies [58]. Surprisingly, fewer than half of the articles presented network maps, which suggests that researchers could do more to elucidate descriptive relational findings for readers. While more complex than descriptive analyses, graphing the results of $\mathrm{p}_{2}$ models can illustrate the relationship between binary network data and covariates, while factoring in network structure [59]. Stacked correspondence analysis of matrices representing different time periods can be used to visualize network data at different time points [7]. Supplemental graphing using conventional visualization methods (e.g., bar, scatterplot, line charts) is also available as an approach to visualize the relationships among network properties and attributes that has yet to be fully leveraged. Appropriate visualization methods and techniques must be selected to answer the research questions of interest, while preserving clarity $[7,60]$.

While a range of analytical techniques was identified, many studies employed traditional analytical techniques designed for data meeting assumptions of independence. By their nature within the SNA paradigm, dyadic data do not meet these assumptions. Techniques designed to account for interdependencies in the data, including quadratic assignment procedure (QAP) analysis and exponential random graph models (ERGM), are considered more robust for those analyses of specific hypotheses involving dyadic ties or network characteristics. These approaches enable the modeling of relationships between dyadic (i.e., relational) variables (e.g., information exchange) and attribute variables (e.g., gender), between dyadic variables (e.g., similarity in EIP attitudes, and engagement in research collaboration), or at the whole network level (e.g., density of communication ties relative to time to evidence adoption) [7]. An example from the included literature is the use of ERGM to help determine whether particular individuals-say those with similar personal characteristics-are connecting for information sharing more than expected due to chance [29].

With the inclusion of longitudinal designs, analysis approaches, such as stochastic actor-based network models (SABM) that examine network change over time, can begin to be represented in this body of literature. SABM can represent both ties and individual attributes to examine network change. As an example, Yousefi-Nooraie et al. [61] used SABM to determine the effect of their intervention (evidence-based decision-making skills) on participants' status as knowledge brokers. 


\section{Network properties}

Further KT-related research that includes analyses of centralization, subgroups, and transitivity may afford a more in-depth understanding of the network-related influences on KT among health professionals. Centralization (i.e., the unevenness of connectivity across the network) can be calculated for the whole network, or for departments or sectors within an organization for the purposes of comparison. Subgroups (e.g., smaller connected groups within a network) can be identified and addressed individually during a KT intervention. For example, isolated individuals can be engaged to form connections with colleagues to benefit from their knowledge or influence. Different subgroups may receive different $\mathrm{KT}$ interventions based on their characteristics and what evidence or theory suggests their influence might be. Efforts to link or to expand subgroups may precede implementation efforts to establish an environment more conducive to change.

Transitivity has been used to examine the tendency of individuals to exchange information with a small versus a large number of sources, and for the network to form highly connected hubs. This analysis can inform the design of KT interventions to improve the efficiency of information sharing or influence (e.g., identifying targets for the intervention and relying on transitive processes to spread the information rather than targeting all network members). Such a strategy can then be compared to alternatives, to test hypotheses about the influence of different network properties on the effectiveness of $\mathrm{KT}$ interventions. This evaluative work is critical to improve our understanding of network influences on KT processes and outcomes.

The range of structural properties examined suggests that researchers consider multiple structural phenomena to be relevant to KT processes and outcomes. Considerable overlap also existed in the real-world phenomena being evaluated by proxy through these properties. This diversity provides a foundation on which to build a stronger knowledge base about networks' multiple influences on research use. This approach aligns closely with current discussion in the KT literature about complex health systems, and the need to use "complexity-informed approaches" to embed evidence-informed changes in the health care system [62-64]. Such systems models assume that health care organizations are dynamic, interdependent, contain sub-systems with feedback loops, and exhibit emergent properties. A combination of a complex adaptive system lens and SNA modeling to measure and explain features of networks and individuals, and most critically the relationship between networks, sub-networks (like cliques), and individuals as they change over time, is an underutilized approach to $\mathrm{KT}$ and implementation. The approach moves from a mechanical understanding of KT barriers and facilitators to a much more complex picture of what is required to introduce and to sustain change in health care organizations.

While complex statistical models are more difficult to apply, they present the benefit of multivariate analyses to better examine the interaction between various factors, as well as the opportunity to control for covariates that may be inflating or masking key effects. As statistical models for SNA continue to emerge, these tools will become increasingly important in clarifying the relative influence of various network properties and attribute effects thought to influence KT.

An understanding of the influences of individual attributes and different types of ties (e.g., friendship) can support the structuring of the health care environment to strengthen network density within homophilous groups (e.g., discipline groups), or to foster greater diversity of collaborations within the network (e.g., interprofessional clinical, project, or professional development work). Knowledge of network structure can be used to target EIP behaviors using a social influence approach that introduces innovation into the network's core or brokers to reduce pervasive information bias, or that facilitates greater density overall. Unsurprisingly, different approaches to KT (e.g., leader-driven vs. collaborative; researcher-led dissemination vs. researcher-clinician collaboration) may present different structural properties (e.g., hierarchical vs. clustering). This finding has implications for information flow, as well as for the development of network interventions to address gaps or to leverage prominent actors in the network to champion the innovation. Those with formal health care leadership roles may not be the only individuals with influence; informal brokers may be more recognizable by network members (particularly peers) as central to KT processes than formal leaders [37, 40]. Alternatively, some networks may not present with prominent central actors or opinion leaders for EIP [29]. A SNA lens can assist in identifying these individuals when they exist, analyzing the extent of their reach, determining the reasons for their prominence, and developing a network-informed plan to leverage their position to advance KT.

Normative group processes and structural position can also explain the timing of adoption, which may be useful in identifying early adopters, opinion leaders, and shared attitudes within a network [23, 24]. Differences reported in the preferred information sources and network influences for early versus late adopters from a diffusion of innovation perspective can be used to guide differential approaches to behaviour change. 


\section{Theoretical insights}

Various theories, frameworks, and models guide KT research efforts [5], although only in a small proportion (3-6\%) of primary research articles in the broader KT field [65, 66]. The included articles demonstrated a broad range of theoretical approaches drawn from the fields of sociology and psychology (e.g., diffusion of innovation, social influence, social contagion, social exchange), as well as from the field of SNA itself (e.g., perspectives that explain the role of weak ties, structural holes, cohesion, or tie homophily on network dynamics). The variety of approaches used suggests that diverse theories may merit exploration for their utility in KT-related SNA research, and that multiple theories may be applied in a single study (e.g., [24, 25, 33]).

Diffusion of innovation theory is applied commonly in the KT literature [5], so its frequent application here was not unexpected. The theory's principles lend themselves well to a SNA paradigm, in that the theory was developed to predict or to explain how information or innovation spreads within social systems [67]. The collection of attribute data about network members permits the analysis of characteristics that influence an individual's adoption of innovation, relative to their network position and other contextual factors [68]. While the majority of KT strategists have adopted an educational approach (i.e., by implementing KT interventions based on an "information dissemination paradigm") [5, 69] to improve awareness, understanding, and attitudes as a means of influencing uptake [70,71], the role of social networks in their impact has yet to be comprehensively studied. Studies that pair traditional educational and behavioral outcomes research with SNA may provide greater insight into the social mechanisms that influence these outcomes.

Social contagion/influence theories are common in the SNA literature, and purport that actors share attitudes, knowledge, or behaviors because of their ties to others who influence them [72]. This perspective highlights the role of peers and others in fostering behavior change, attitudes, and identities [69]. The study of opinion leaders, audit and feedback [5, 66, $71]$, and mentoring $[73,74]$ may follow a social influence perspective [69], as does a growing body of research on knowledge brokering as a human mediator of research uptake in health care [75]. Social capital theory, another prominent SNA theory, attributes tie formation to an individual's need for social capital from others (e.g., resources, information, power). This contrasting approach was used in the context of knowledge brokering; its application in tandem with social influence theory may provide insight into the direction of causality (i.e., ties form because of attributes vs. attributes are the result of ties) [7].
Transactive memory theory, social exchange theory, Habermas' theory of communicative power, sociology of professions theory, balance theory, and an epistemic differences perspective have logical applications to SNA. Each of these perspectives addresses social factors applicable to networks. The transactive memory-social exchange model describes how information seeking behavior is influenced by the awareness and valuing of another individual's knowledge or skills, their accessibility, and the cost or effort involved in seeking the information [37]. Communicative power is meant to describe the influence of a third party on the mutual understanding achieved between a pair of individuals [42]. Sociology of professions theory describes how members of a shared profession tend to develop a collective status based on their unique health care jurisdictions, common training, and mutual knowledge, which can limit their tendency to interact outside this peer group [22]. Balance theory contends that individuals tend to develop balanced relationships (e.g., tie reciprocity) in order to circumvent unease [27]. An epistemic differences perspective purports that individual attributes in combination with structures, processes, and other features of the environmental context (including power), influence individual performance and experiences [39]. These differences create diversity within the network that generate opportunities for novel sharing and innovation [39]. By understanding the structural network factors that contribute to information seeking, epistemic differences, power dynamics, and communication processes, we may gain a more holistic understanding of KT. Further analysis is required to determine the utility other theories and frameworks from the $\mathrm{KT}$ and SNA literature to SNA-related KT research.

The study of networks is particularly relevant when considering the role of social evaluation in the diffusion of innovation. Gartrell [76] argues that social evaluation is a key driver of decision-making, noting the role of networks in providing norms and comparison opportunities that influence behavior. Social influence theory, and the principles of tie homophily, social contagion, and structural equivalence parallel this line of thought. While the role of context is addressed relatively commonly in KT and implementation models and frameworks [75], the concept of social evaluation is largely absent from the non-SNA KT literature. SNA can be applied to identify the attributes, structural positions, or nature of the relationships that are most influential for $\mathrm{KT}$ processes from a social evaluation perspective, including the role of brokers, homophily, hierarchy, centrality, and other network properties. These network characteristics offer insights into the patterns and restrictions in the flow of information as well as into power 
structures, key actors, and their reach and efficiency. Normative group processes and structural position can also explain the timing of adoption, which may be useful in identifying early adopters, opinion leaders, and the development of shared attitudes within a network $[24,76]$.

Social constructivist and cognitive learning theories have also emerged in the KT literature to explore the means by which health professionals interact within their social context to construct and to understand knowledge $[66,77,78]$. The network perspective has not been combined with these approaches to enrich our understanding of the role of this social context on evidence uptake. Also missing from the included articles is reference to behavior change theories that have been prevalent in the KT literature [5]. These theories emphasize the various barriers and facilitators to change, including personal, organizational, and system-level factors [65]. Integrating a SNA lens to behavior change models may augment their utility by including social structure as a determinant of behaviour change.

\section{Limitations}

The search and screening process used in this review limited inclusion to studies involving quantitative analysis of SNA data; a greater depth of understanding about SNA's utility for KT may also be gained from theoretical discussion papers. Also omitted was the contribution of qualitative data from network research, which can provide insight into how social structures, network properties, and the nature of relationships influence KT from the perspectives of network members. A broader set of inclusion criteria that encompassed studies on the adoption of non-clinical best practices, including technologies, training strategies, quality improvement initiatives and other innovations, as well as KT in the policy context, may also inform aspects of KT research and implementation initiatives, but was beyond the scope of this review.

This discussion only addressed these studies' SNA-specific findings. Important non-SNA-related findings (e.g., the most important sources of evidence to augment awareness and adoption [23]) have not been summarized but can also inform KT research and practice. In addition, the scope of this paper prevented an in-depth discussion of the full breadth of theoretical perspectives represented in the SNA KT literature, which may warrant a separate review. Finally, because of the variability in study designs, the lack of inclusion of quality appraisals in scoping review methodology, and the inconsistency of network properties examined across the studies, results should be interpreted with caution until further research can evaluate their quality and generalizability.

\section{Conclusions}

Given the diversity and complexity of health professional KT networks, optimal strategies for KT may vary depending on the structure of a professional or organizational network, as well as on professional identities and personal attributes. Within a given setting, interprofessional dynamics, hierarchy, social influence, centralization, brokers, and other important structural properties are worthy of consideration. The SNA paradigm offers a broader lens by which to examine and to influence KT processes and outcomes across contexts, while drawing on established theories known to the KT science field. SNA extends the scope of KT influence to include social relationships and structural characteristics of individual and whole networks. However, its full potential has yet to be realized.

Suitable for relatively small (e.g., a dozen) to larger networks of several hundred members or more, SNA can be used to describe or to evaluate groups within or across departments, organizations, countries, or beyond. Longitudinal research, a more representative range of populations, the use of interviews, document review and observation for data collection, greater depth of analysis, and the leveraging of network visualizations can augment the contributions of SNA to the KT science knowledge base.

Understanding how network properties can be used as proxies to measure social processes (e.g., information exchange, best practice adoption, decision-making, influence) can help KT scientists to apply SNA effectively to expand the range of measures that can be used to evaluate KT efforts. The approach can be used to describe a network as a precursor to a $\mathrm{KT}$ intervention, as a means of supporting planning (e.g., identifying target groups or individuals), as well as for testing hypotheses. Evaluating information sharing, positions of influence, relationships between network connection patterns and individual attributes (e.g. attitudes) or behaviors, and the effectiveness of KT interventions relying on or targeting networks are all feasible. Predicting or explaining patterns of connections, comparing groups, time points or contexts are also possible.

Finally, while this article did not present a comprehensive overview of the use of theory across the entire body of SNA-related KT literature, it does offer a starting point for conceptualizing theory-based SNA applications in KT research. In keeping with a systems or complexity theory approach, SNA can offer a wider spectrum of determinants to examine in evaluating $\mathrm{KT}$ processes by addressing social factors.

The targeted SNA research outlined here may help to highlight the role of interactions, relationships, and other social dynamics throughout the full scope of activities and processes required to move evidence into action within health care settings and beyond. 


\section{Appendix}

Table 4 OvidSP MEDLINE search strategy

\begin{tabular}{lll}
\hline Concept & Keywords & MeSH Headings \\
\hline Health care & Clinician* OR "health care professiona**" OR & Allied Health Personnel OR Medical \\
professionals & "health professiona*" OR therapis** OR physician* & Staff Hospital
\end{tabular}

Knowledge Knowledge translation OR evidence based practice

translation OR evidence informed practice OR dissemination

OR organi*ational innovation OR implementation adj3 research OR research utili*ation OR research use OR knowledge utili*ation OR knowledge transfer OR knowledge mobili*ation OR knowledge exchange

Social network analysis
Knowledge Management OR Translational Medical Research OR Diffusion of Innovation OR Evidence-Based Practice OR Professional Practice OR Guideline Adherence OR Social Change

Interprofessional Relations

\section{Abbreviations}

CINAHL: Cumulative Index to Nursing and Allied Health Literature; EIP: Evidence informed practice; KT: Knowledge translation; SIENA: Simulation investigation for empirical network analysis; SNA: Social network analysis

\section{Acknowledgments}

The authors would like to acknowledge the contributions of Dr. David Tindall in helping to shape the refinement of a previous version of this manuscript, and Andrea Ryce for her contributions to the development of the search strategy.

\section{Funding}

SG was supported by a Vanier Canada Graduate Scholarship, a Canadian Child Health Clinician Scientist Program Career Enhancement Award, a FourYear Fellowship, Faculty of Medicine Graduate Award, Rehabilitation Sciences Doctoral Scholarship and Public Scholars Award from the University of British Columbia, and funding from the Sunny Hill Foundation.

\section{Availability of data and materials}

All data generated or analyzed during this study are included in this published article.

\section{Authors' contributions}

SG designed the study, conducted the search, screened for inclusion, extracted data, synthesized the literature, and drafted the manuscript. EJ screened for inclusion, contributed to study design refinement and revisions to draft manuscript. AK verified data extraction and contributed to study design refinement and revisions to draft manuscript. All authors read and approved the final manuscript.

\section{Authors' information}

SG is a doctoral candidate in Rehabilitation Sciences at the University of British Columbia, and the Knowledge Broker Facilitator with the Child Development \& Rehabilitation Evidence Centre at Sunny Hill Health Centre for Children. EJ is an Assistant Professor in the School of Nursing at the University of British Columbia. AK is an Associate Professor in the School of Health Studies at Western University.

\section{Ethics approval and consent to participate} Not applicable.

\section{Consent for publication}

Not applicable.

\section{Competing interests}

The authors declare that they have no competing interests. This study represents work conducted in partial fulfillment of SG's doctoral thesis.

\section{Publisher's Note}

Springer Nature remains neutral with regard to jurisdictional claims in published maps and institutional affiliations.

\section{Author details}

${ }^{1}$ Rehabilitation Sciences, The University of British Columbia, 212 - 2177 Wesbrook Mall, Vancouver, BC V6T 1Z3, Canada. ${ }^{2}$ Therapy Department, Sunny Hill Health Centre for Children, 3644 Slocan Street, Vancouver, BC V5M 3E8, Canada. ${ }^{3} \mathrm{BC}$ Children's Hospital Research Institute, 938 West 28th Ave, Vancouver, BC V5Z 4H4, Canada. ${ }^{4}$ School of Nursing, The University of British Columbia, T201-2211 Wesbrook Mall, Vancouver, BC V6T 2B5, Canada. ${ }^{5}$ School of Health Studies, Western University, Arthur and Sonia Labatt Health Sciences Building, Room 222, London, ON V6A 5B9, Canada.

Received: 17 August 2018 Accepted: 11 March 2019

Published online: 27 March 2019

\section{References}

1. Graham ID, Tetroe J. Some theoretical underpinnings of knowledge translation. Acad Emerg Med. 2007;14:936-41.

2. Menon A, Korner-Bitensky N, Kastner M, McKibbon KA, Straus S. Strategies for rehabilitation professionals to move evidence-based knowledge into practice: a systematic review. J Rehabil Med. 2009;41(13):1024-32. [cited 2015 Aug 18]. Available from: https://medicaljournals.se/jrm/content/ abstract/10.2340/16501977-0451.

3. Nilsen P. Making sense of implementation theories, models and frameworks. Implement Sci. 2015;10(1):53.

4. LaRocca R, Yost J, Dobbins M, Ciliska D, Butt M. The effectiveness of knowledge translation strategies used in public health: a systematic review. BMC public health. 2012;12(1):751. Available from: BMC Public Health

5. Colquhoun HL, Letts LJ, Law MC, MacDermid JC, Missiuna CA. A scoping review of the use of theory in studies of knowledge translation. Can J Occup Ther. 2010;77(5):270-9.

6. Chambers D, Wilson P, Thompson C, Harden M. Social network analysis in healthcare settings: a systematic scoping review. PLoS One. 2012;7(8): e41911.

7. Borgatti SP, Everett MG, Johnson JC. Analyzing social networks. Los Angeles: SAGE Publications; 2013. p. 296.

8. Cunningham FC, Ranmuthugala G, Plumb J, Georgiou A, Westbrook J, Braithwaite J. Health professional networks as a vector for improving healthcare quality and safety: a systematic review. BMJ Qual Saf. 2012;21(3): 239-49.

9. Bae S, Nikolaev A, Seo JY, Castner J. Health care provider social network analysis: a systematic review. Nurs Outlook. 2015;63(5):566-84.

10. Benton DC, Perez-Raya F, Fernandez-Fernandez MP, Gonzalez-Jurado MA. A systematic review of nurse-related social network analysis studies. Int Nurs Rev. 2015;62(3):321-39.

11. Hurlock-Chorostecki C, Forchuk C, Orchard C, van Soeren M, Reeves S. Hospital-based nurse practitioner roles and interprofessional practice: a scoping review. Nurs Health Sci. 2014;16:403-10.

12. Zhang S, de la Haye K, Ji M, An R. Applications of social network analysis to obesity: a systematic review. Obes Etiol. 2018;19(7):976-88.

13. Long JC, Cunningham FC, Braithwaite J, Braithwaite J, Braithwaite J, Westbrook $\mathrm{M}$, et al. Bridges, brokers and boundary spanners in collaborative networks: a systematic review. BMC Health Serv Res. 2013;13(1):158. 
14. Tasselli S. Social networks of professionals in health care organizations: a review. Med Care Res Rev. 2014;71(6):619-60.

15. Sabot K, Wickremasinghe D, Blanchet K, Avan B, Schellenberg J. Use of social network analysis methods to study professional advice and performance among healthcare providers: a systematic review. Syst Rev. 2017;6:208.

16. Arksey H, O'Malley L. Scoping studies: towards a methodological framework. Int J Soc Res Methodol. 2005:8(1):19-32

17. Levac D, Colquhoun H, O'Brien KK. Scoping studies: advancing the methodology. Implement Sci. 2010;5(1):69.

18. KA MK, Lokker C, Wilczynski NL, Haynes RB, Ciliska D, Dobbins M, et al. Search filters can find some but not all knowledge translation articles in MEDLINE: an analytic survey. J Clin Epidemiol. 2012;65:651-9.

19. McKibbon KA, Lokker C, Wilczynski NL, Ciliska D, Dobbins M, Davis DA, et al. Cross-sectional study of the number and frequency of terms used to refer to knowledge translation in a body of health literature in 2006: a tower of babel? Implement Sci. 2010;5(1):16.

20. Lokker C, McKibbon KA, Wilczynski NL, Haynes RB, Ciliska D, Dobbins M, et al. Finding knowledge translation articles in CINAHL. Stud Health Technol Inform. 2010;160(Pt 2):1179-83.

21. Sibbald SL, Wathen CN, Kothari A, Day AMB. Knowledge flow and exchange in interdisciplinary primary health care teams (PHCTs): An exploratory study. J Med Libr Assoc. 2013;101(2):128-37. Available from: https://www-ncbinlm-nih-gov.ezproxy.library.ubc.ca/pmc/articles/PMC3634376/.

22. Tasselli S. Social networks and inter-professional knowledge transfer: the case of healthcare professionals. Organ Stud. 2015;36(7):841-72.

23. Ankem K. Influence of information sources on the adoption of uterine fibroid embolization by interventional radiologists. J Med Libr Assoc. 2003; 91(4):450-9.

24. Burt RS. Social contagion and innovation: cohesion versus structural equivalence. Am J Sociol. 1987;92(6):1287-335.

25. Friedkin NE. A multilevel event history model of social diffusion: medical innovation revisited. J Math Sociol. 2010;34(2):146-55. Available from: https://www.tandfonline.com/doi/abs/10.1080/00222500903221589,

26. Heijmans $N$, van Lieshout J, Wensing M. Information exchange networks of health care providers and evidence-based cardiovascular risk management: an observational study. Implement Sci [Internet]. 2017;12(1):1-12. Available from: https://doi.org/10.1186/s13012-016-0532-1

27. Mascia D, Pallotti F, Dandi R. Determinants of knowledge-sharing networks in primary care. Health Care Manag Rev. 2018;43(2):104-14.

28. Di Vincenzo F, Mascia D. Knowledge development and advice networks in professional organizations. Knowl Manag Res Pract. 2017;15(2):201-13.

29. Zappa P. The network structure of knowledge sharing among physicians. Qual Quant. 2011;45(5):1109-26. Available from: https://healthcouncilcanada. ca/files/2.48-2006_AnnualReport_1.pdf.

30. Mascia D, Cicchetti A, Fantini MP, Damiani G, Ricciardi W. Physicians' propensity to collaborate and their attitude towards EBM: a cross-sectional study. BMC Health Serv Res. 2011;11(1):172.

31. Mascia D, Cicchetti A. Physician social capital and the reported adoption of evidence-based medicine: exploring the role of structural holes. Soc Sci Med. 2011;72(5):798-805. Available from: https://doi.org/10.1016/j.socscimed. 2010.12.011

32. Mascia D, Cicchetti A, Damiani G. "Us and Them": a social network analysis of physicians' professional networks and their attitudes towards EBM. BMC Health Serv Res. 2013;13(1):429.

33. Mascia D, Dandi R, Di Vincenzo F. Professional networks and EBM use: a study of inter-physician interaction across levels of care. Health Policy (New York). 2014;118(1):24-36.

34. Doumit GD, Papay FA, Moores N, Meisler E, Zins JE. Opinion leaders and evidence-based medicine in craniofacial surgery. J Craniofac Surg. 2014; 25(1):106-10.

35. Paul S, Keating NL, Landon BE, O'Malley AJ. Reprint of: results from using a new dyadic-dependence model to analyze sociocentric physician networks. Soc Sci Med. 2015;125:51-9. Available from: https://doi.org/10.1016/j. socscimed.2014.08.027

36. Yousefi-Nooraie R, Dobbins M, Brouwers M, Wakefield P. Information seeking for making evidence-informed decisions: a social network analysis on the staff of a public health department in Canada. BMC Health Serv Res. 2012;12:118.

37. Yousefi-Nooraie R, Dobbins M, Marin A. Social and organizational factors affecting implementation of evidence-informed practice in a public health department in Ontario: a network modelling approach. Implement Sci. 2014;9:29. Available from: https://implementationscience.biomedcentral. com/articles/10.1186/1748-5908-9-29.

38. Guldbrandsson K, Nordvik MK, Bremberg S. Identification of potential opinion leaders in child health promotion in Sweden using network analysis. BMC Res Notes. 2012;5:42. https://doi.org/10.1186/1756-0500-5-424, https://www.ncbi.nlm.nih.gov/pubmed/22873749.

39. D'Andreta D, Scarbrough H, Evans S. The enactment of knowledge translation: a study of the Collaborations for Leadership in Applied Health Research and Care initiative within the English National Health Service. J Health Serv Res Policy. 2013;18(Suppl 3):40-52.

40. Long JC, Cunningham FC, Carswell P, Braithwaite J. Who are the key players in a new translational research network? BMC Health Serv Res. 2013;13:1.

41. Long JC, Cunningham FC, Carswell P, Braithwaite J. Patterns of collaboration in complex networks: the example of a translational research network. BMC Health Serv Res. 2014;14(1):225. Available from: https://bmchealthservres. biomedcentral.com/articles/10.1186/1472-6963-14-225.

42. Quinlan E, Robertson S. The communicative power of nurse practitioners in multidisciplinary primary healthcare teams. J Am Assoc Nurse Pract. 2013: 25(2):91-102.

43. Bunger AC, Hanson RF, Doogan NJ, Powell BJ, Cao Y, Dunn J. Can learning collaboratives support implementation by rewiring professional networks? Adm Policy Ment Health. 2016;43(1):79-92. Available from: https://doi.org/10. 1007/s10488-014-0621-x

44. Menchik DA, Meltzer DO, Menchik DA, Meitzer DO. The cultivation of esteem and retrieval of scientific knowledge in physician networks. J Health Soc Behav. 2017;51(2):137-52.

45. Keating NL, Ayanian JZ, Cleary PD, Marsden PV. Factors affecting influential discussions among physicians: a social network analysis of a primary care practice. J Gen Intern Med. 2007;22(6):794-8.

46. Graham ID, Logan J, Harrison MB, Straus SE, Tetroe J, Caswell W, et al. Lost in knowledge translation: time for a map? J Contin Educ Heal Prof. 2006; 26(1):13-24.

47. Racko G. Knowledge exchange in the UK CLAHRCs: the enabling role of academics and clinicians' social position. J Health Organ Manag. 2018;32(2): 246-62.

48. Mascia D, Di Vincenzo F, lacopino V, Fantini MP, Cicchetti A. Unfolding similarity in interphysician networks: the impact of institutional and professional homophily. BMC Health Serv Res. 2015;15(1):1-9.

49. Valente TW, Pumpuang P. Identifying opinion leaders to promote behavior change. Heal Educ Behav. 2007;34(6):881-96. Available from: http://journals. sagepub.com/doi/10.1177/1090198106297855

50. Valente TW, Fujimoto K. Bridging: locating critical connectors in a network. Soc Networks. 2010;32(3):212-20. Available from: https://doi.org/10.1016/j. socnet.2010.03.003

51. Valente TW, Fujimoto K, Palmer P, Tanjasiri SP. A network assessment of community-based participatory research: linking communities and universities to reduce cancer disparities. Am J Public Health. 2010;100(7): 1319-25.

52. Valente TW, Palinkas LA, Czaja S, Chu K, Brown CH. Social network analysis for program implementation 2015;1-18. Available from: https://doi.org/10. 1371/journal.pone.0131712

53. Valente TW. Network Interventions. Science. 2012;337(6090):49-54.

54. Snijders TAB. The SIENNA webpage [Internet]. [cited 2017 Nov 28]. Available from: https://www.stats.ox.ac.uk/ snijders/siena/. Accessed 15 July 2018.

55. El-Sayed AM, Scarborough P, Seemann L, Galea S. Social network analysis and agent-based modeling in social epidemiology. Epidemiol Perspect Innov. 2012;9(1):1.

56. Hollstein B. Mixed methods social networks research: an introduction. In: Dominguez S, Hollstein B, editors. Mixed methods social networks research: design and applications. New York: Cambridge University Press; 2014. p. 3-33.

57. Health Council Canada. Health care renewal in Canada: Clearing the road to quality. Annual report to Canadians [Internet]. Eglinton, ON; 2005. Available from: http://healthcouncilcanada.ca/accord_theme.php?mnu=2\&mnu1= $36 \&$ mnu2 $=29$. Accessed 15 July 2018

58. Glegg SMN. Facilitating interviews in qualitative research with visual tools: a typology. Qual Health Res. 2018;29(2):301-10. https://journals.sagepub.com/ doi/abs/10.1177/1049732318786485.

59. van Duijn MAJ, Snijders TAB, Zijlstra BJH. P2: A random effects model with covariates for directed graphs. Stat Neerl. 2004;58(2):234-54. 
60. Knoke D, Yang S. Social network analysis. 2nd ed. Thousand Oaks: SAGE Publications; 2008. $133 \mathrm{p}$.

61. Yousefi-nooraie R, Dobbins M, Marin A, Hanneman R, Lohfeld L. The evolution of social networks through the implementation of evidenceinformed decision-making interventions: a longitudinal analysis of three public health units in Canada. Implement Sci. 2015;10:166. Available from: https://doi.org/10.1186/s13012-015-0355-5.

62. Best A, Holmes B. Systems thinking, knowledge and action: towards better models and methods. Evid Policy A J Res Debate Pract. 2010;6(1):145-59.

63. Holmes BJ, Best A, Davies H, Hunter D, Kelly MP, Marshall M, et al. Mobilising knowledge in complex health systems: a call to action. Evid Policy A J Res Debate Pract. 2017;13(3):539-60.

64. Braithwaite J, Churruca K, Long JC, Ellis LA, Herkes J. When complexity science meets implementation science: a theoretical and empirical analysis of systems change. BMC Med. 2018;16:63.

65. Michie S, van Stralen MM, West R, Grimshaw J, Shirran L, Thomas R, et al. The behaviour change wheel: a new method for characterising and designing behaviour change interventions. Implement Sci. 2011;6(1):42. Available from: http://implementationscience.biomedcentral.com/articles/10. 1186/1748-5908-6-42

66. Colquhoun HL, Brehaut JC, Sales A, Ivers N, Grimshaw J, Michie S, et al. A systematic review of the use of theory in randomized controlled trials of audit and feedback. Implement Sci. 2013;8(1):66.

67. Rogers E. Diffusion of innovations. New York: Free Press; 1995

68. Greenhalgh T, Robert G, Macfarlane F, Bate P. Diffusion of innovations in service organizations: systematic review and recommendations. Milbank Q 2004;82(4):581-629.

69. Mano-Negrin R, Mittman B. Theorising the social within physician decision making. J Manag Med. 2011;15(4-5):663-7.

70. Yamada J, Shorkey A, Barwick M, Widger K, Stevens BJ. The effectiveness of toolkists as knowledge translation strategies for integrating evidence into clinical care: a systematic review. BMJ Open. 2015;5(4):e006808.

71. Jones CA, Roop SC, Pohar SL, Albrecht L, Scott SD. Translating knowledge in rehabilitation: systematic review. Phys Ther. 2015;95(4):663-7.

72. Friedkin NE. A structural theory of social influence. Structural analysis in the social sciences. New York: Cambridge University Press; 1998.

73. Gagliardi AR, Fiona W, Perrier L, Bell M, Straus S. Exploring mentorship as a strategy to build capacity for knowledge translation research and practice: a scoping systematic review. Implement Sci. 2014;9(1):122.

74. Abdullah G, Rossy D, Ploeg J, Davies B, Higuchi K, Sikora L, et al. Measuring the effectiveness of mentoring as a knowledge translation intervention for implementing empirical evidence: a systematic review. Worldviews Evidence-Based Nurs. 2014;11(5):284-300.

75. Glegg SMN, Hoens AM. The role domains of knowledge brokering: a model for the healthcare setting. J Neurol Phys Ther. 2016;40(2):115-23.

76. Gartrell CD. Network approaches to social evaluation. Annu Rev Sociol. 1987;13:49-66.

77. Thomas A, Menon A, Boruff J, Rodriguez AM, Ahmed S. Applications of social constructivist learning theories in knowledge translation for healthcare professionals: a scoping review. Implement Sci. 2014;9(1):54.

78. Brehaut JC, Eva KW. Building theories of knowledge translation interventions: use the entire menu of constructs. Implement Sci. 2012;

79. Hanneman RA, Riddle M. Introduction to social network methods [internet]. Riverside: University of California, Riverside; 2005. 332 p. Available from: http://faculty.ucr.edu/ hanneman/nettext/.

80. Baldassarri D, Diani M. The integrative power of civic networks. Am J Sociol. 2007:113:735-80

81. Burt RS. Structural holes. In: structural holes. Cambridge: Cambridge University Press; 1992.

82. Granovetter MS. The strength of weak ties. Am J Sociol. 1973;78(6):1360-80.

83. Coleman JS. Social capital in the creation of human capital. Am J Sociol. 1988;94:595-120.

84. West E, Barron DN, Dowsett J, Newton JN. Hierarchies and cliques in the social networks of health care professionals: implications for the design of dissemination strategies. Soc Sci Med. 1999;48(5):633-46.

\section{Ready to submit your research? Choose BMC and benefit from:}

- fast, convenient online submission

- thorough peer review by experienced researchers in your field

- rapid publication on acceptance

- support for research data, including large and complex data types

- gold Open Access which fosters wider collaboration and increased citations

- maximum visibility for your research: over $100 \mathrm{M}$ website views per year

At BMC, research is always in progress.

Learn more biomedcentral.com/submissions 